\title{
ON VALIDITY OF THE ASYMPTOTIC EXPANSION APPROACH IN CONTINGENT CLAIM ANALYSIS
}

\author{
By NAOTO KUNITOMO AND AKIHIKO TAKAHASHI \\ University of Tokyo
}

\begin{abstract}
Kunitomo and Takahashi $(1995,2001)$ have proposed a new methodology, called small disturbance asymptotics, for the valuation problem of financial contingent claims when the underlying asset prices follow a general class of continuous Itô processes. It can be applicable to a wide range of valuation problems, including complicated contingent claims associated with the Black-Scholes model and the term structure model of interest rates in the Heath-Jarrow-Morton framework. Our approach can be rigorously justified by an infinite-dimensional analysis called the Watanabe-Yoshida theory on the Malliavin calculus recently developed in stochastic analysis.
\end{abstract}

1. Introduction. In the past decades various contingent claims including futures, options, swaps and other derivative securities have been introduced and actively traded in financial markets. Except some simple cases such as the original Black-Scholes model in which the underlying assets follow the geometric Brownian motions and the risk free rate is constant, however, it has been difficult to derive the explicit formulas for the fair market values of financial contingent claims. Meanwhile, Kunitomo and Takahashi (1995, 2001), and Takahashi (1999) have presented a new methodology called the small disturbance asymptotic theory which is widely applicable to the valuation problem of financial contingent claims when the underlying asset prices follow the general class of continuous Itô processes. They have given rather simple formulas which are useful for various valuation problems of contingent claims in financial economics.

For the Black-Scholes economy, Takahashi (1999) has systematically investigated the valuation problem of various contingent claims when the vector of $d$ asset prices $S_{t}=\left(S_{t}^{i}\right)(i=1, \ldots, d ; 0 \leq t \leq T<+\infty)$ follows the stochastic differential equation

$$
S_{t}^{i}=S_{0}^{i}+\int_{0}^{t} \mu_{*}^{i}\left(S_{v}, v\right) d v+\sum_{j=1}^{m} \int_{0}^{t} \sigma_{*}^{i j}\left(S_{v}, v\right) d w_{v}^{j},
$$

where $d \times 1$ vector $\mu_{*}\left(S_{v}, v\right)=\left(\mu_{*}^{i}\left(S_{v}, v\right)\right)$ and $d \times m$ matrix $\sigma_{*}\left(S_{v}, v\right)=$ $\left(\sigma_{*}^{i j}\left(S_{v}, v\right)\right)$ are the instantaneous mean and the volatility functions, respectively, and $\left\{w_{v}^{i}\right\}$ are Brownian motions. In this Black-Scholes economy, we have to

Received May 2000; revised February 2002.

AMS 2000 subject classifications. Primary 90A09; secondary 60H07.

Key words and phrases. Valuation of financial contingent claims, asymptotic expansion, small disturbance asymptotics, validity, Watanabe-Yoshida theory, Malliavin calculus. 
change the underlying measure because of the no-arbitrage theory in finance [see, e.g., Chapter 6 of Duffie (1996) on the standard theory]. Then we can consider the situation when $S_{t}^{(\varepsilon)}$ satisfies

$$
S_{t}^{(\varepsilon)}=S_{0}+\int_{0}^{t} r\left(S_{v}^{(\varepsilon)}, v\right) S_{v}^{(\varepsilon)} d v+\varepsilon \int_{0}^{t} \sigma\left(S_{v}^{(\varepsilon)}, v\right) d w_{v},
$$

where $S_{t}^{(\varepsilon)}\left[=\left(S_{t}^{(\varepsilon) i}\right)\right]$ is a $d \times 1$ vector with the parameter $\varepsilon(0<\varepsilon \leq 1)$, $\sigma\left(S_{v}^{(\varepsilon)}, v\right)(d \times m)$ is the volatility term, $r(\cdot, \cdot)$ is the risk free (positive) interest rate, and $w_{v}\left[=\left(w_{v}^{i}\right)\right]$ is an $m \times 1$ vector. The small disturbance asymptotic theory under the no-arbitrage theory can be constructed by considering the situation when $\varepsilon \rightarrow 0$ and we can develop the valuation method of contingent claims based on $\left\{S_{t}^{(\varepsilon)}\right\}$.

Note. The limit of stochastic process $S_{t}^{(\varepsilon)}$ is the solution of an ordinary differential equation when $\varepsilon \rightarrow 0$ in this formulation. There can be an alternative formulation such that the limit is the solution of a stochastic differential equation. See Kim and Kunitomo (1999), Kunitomo and Kim (2001), Sørensen and Yoshida (2000) or Takahashi and Yoshida (2001) on this formulation and some applications in financial problems. However, it requires a set of different arguments including the partial Malliavin covariances.

For the term structure model of interest rates in the HJM framework [Heath, Jarrow, and Morton (1992)], let $P(s, t)$ denote the price of the discount bond at $s$ with maturity date $t(0 \leq s \leq t \leq T<+\infty)$. When it is continuously differentiable with respect to $t$ and $P(s, t)>0$ for $0 \leq s \leq t \leq T$, the instantaneous forward rate at $s$ for future date $t(0 \leq s \leq t \leq T)$ is given by $f(s, t)=-\frac{\partial \log P(s, t)}{\partial t}$. The no-arbitrage condition requires the drift restrictions on a family of forward rates processes $\{f(s, t)\}$ for $0 \leq s \leq t \leq T$ to follow the stochastic integral equation

$$
\begin{aligned}
f(s, t)= & f(0, t) \\
& +\int_{0}^{s} \sum_{i=1}^{m}\left[\sigma_{*}^{i}(f(v, t), v, t) \int_{v}^{t} \sigma_{*}^{i}(f(v, y), v, y) d y\right] d v \\
& +\sum_{i=1}^{m} \int_{0}^{s} \sigma_{*}^{i}(f(v, t), v, t) d w_{v}^{i},
\end{aligned}
$$

where $f(0, t)$ are nonrandom initial forward rates, $\left\{w_{v}^{i} ; i=1, \ldots, m\right\}$ are $m$ Brownian motions and $\left\{\sigma_{*}^{i}(f(v, t), v, t) ; i=1, \ldots, m\right\}$ are the volatility functions. When $f(s, t)$ is continuous at $s=t$ for $0 \leq s \leq t \leq T$, the instantaneous spot interest rate process can be defined by $r(t)=\lim _{s \rightarrow t} f(s, t)$. In this framework of stochastic interest rate economy, Kunitomo and Takahashi (1995, 2001) have investigated the valuation of contingent claims when a family of 
forward rate processes obey

$$
\begin{aligned}
f^{(\varepsilon)}(s, t)= & f(0, t) \\
& +\varepsilon^{2} \int_{0}^{s} \sum_{i=1}^{m}\left[\sigma^{i}\left(f^{(\varepsilon)}(v, t), v, t\right) \int_{v}^{t} \sigma^{i}\left(f^{(\varepsilon)}(v, y), v, y\right) d y\right] d v \\
& +\varepsilon \sum_{i=1}^{m} \int_{0}^{s} \sigma^{i}\left(f^{(\varepsilon)}(v, t), v, t\right) d w_{v}^{i},
\end{aligned}
$$

where $0<\varepsilon \leq 1$. The volatility functions $\left\{\sigma^{i}\left(f^{(\varepsilon)}(s, t), s, t\right) ; i=1, \ldots, m\right\}$ depend not only on $s$ and $t$, but also on $f^{(\varepsilon)}(s, t)$ in the general case. The instantaneous spot interest rate process can be defined by $r^{(\varepsilon)}(t)=\lim _{s \rightarrow t} f^{(\varepsilon)}(s, t)$. Then the small disturbance asymptotic theory can be constructed by considering the situation when $\varepsilon \rightarrow 0$ and we can develop the valuation method of contingent claims based on $\left\{f^{(\varepsilon)}(s, t)\right\}$ and the discount bond prices

$$
P^{(\varepsilon)}(t, T)=\exp \left[-\int_{t}^{T} f^{(\varepsilon)}(t, u) d u\right] .
$$

The main purpose of this paper is to give the validity of the asymptotic expansion approach along the line called the Watanabe-Yoshida theory on the Malliavin calculus recently developed in stochastic analysis. The Malliavin calculus has been developed as an infinite-dimensional analysis of Wiener functionals by several probablists in the last two decades. We intend to apply this powerful calculus on continuous stochastic processes to the valuation problem of financial contingent claims along the line developed by Watanabe (1987) and subsequently by Yoshida (1992). However, the continuous-time stochastic processes appearing in financial economics are not necessarily time-homogeneous Markovian in the usual sense while the existing asymptotic expansion methods initiated by Watanabe (1987) and refined by Yoshida (1992) have been developed for time-homogeneous Markovian processes. Hence we need to extend some of the existing results on the validity of the asymptotic expansion approach. Also the mathematical devices used in the Watanabe-Yoshida theory have not been standard for finance as well as in many applied fields due to the recent mathematical developments involved. In this paper we intend to give a rigorous discussion on the validity of the asymptotic expansion approach in a unified way. Although some of the following derivations have been already reported in Kunitomo and Takahashi (1995, 2001) and Takahashi (1999), these papers did not give many important proofs on the validity of the asymptotic expansion approach.

In this paper we shall also illustrate the usefulness of the asymptotic expansion approach by showing some numerical examples. Since several related papers have already appeared [Kunitomo and Takahashi (2001) and Takahashi (1999), e.g.], we shall only discuss simple examples with analytical difficulties from other approaches. 
In Section 2, we give some preliminary mathematical devices, that will be needed in the following derivations. Section 3 is on the validity of our approach for the continuous Markovian setting, while Section 4 is on the validity of our approach for the HJM setting of the interest rates model. We give some numerical examples in Section 5 and concluding remarks in Section 6. Some mathematical details will be given in the Appendix.

2. Preliminary mathematics. We shall first prepare the fundamental results, including Theorem 2.2 of Yoshida (1992), which is in turn a truncated version of Theorem 2.3 of Watanabe (1987). The theory by Watanabe (1987) on the Malliavin calculus and Theorem 2.2 of Yoshida (1992) are the fundamental ingredients to show the validity of our asymptotic expansion method. This is the reason why we call it the Watanabe-Yoshida theory on the Malliavin calculus. For our purpose, we shall freely use the notation by Ikeda and Watanabe (1989) as a standard textbook. The interested reader should see Watanabe (1984, 1987), Ikeda and Watanabe (1989), Yoshida (1992, 1997), Shigekawa (1998) or Nualart (1995).

2.1. Some notation and definitions. Let $\boldsymbol{W}$ be the $m$-dimensional Wiener space, which is a Banach space consisting of the totality of continuous functions $w:[0, T] \rightarrow R^{m}[w(0)=0]$ with the topology induced by the norm $\|w\|=$ $\max _{0 \leq t \leq T}|w(t)|$. Let also $\boldsymbol{H}$ be the Cameron-Martin subspace of $\boldsymbol{W}$, where $h(t)=\left(h^{j}(t)\right) \in \boldsymbol{H}$ is in $\boldsymbol{W}$ and is absolutely continuous on $[0, T]$ with square integrable derivative $\dot{h}(t)$ endowed with the inner product defined by

$$
\left\langle h_{1}, h_{2}\right\rangle_{H}=\sum_{j=1}^{m} \int_{0}^{T} \dot{h}_{1}^{j}(s) \dot{h}_{2}^{j}(s) d s .
$$

We shall use the notation of the $H$-norm as $|h|_{H}^{2}=\langle h, h\rangle_{H}$ for any $h \in \boldsymbol{H}$. A function $f: \boldsymbol{W} \mapsto \boldsymbol{R}$ is called a polynomial functional if there exist $n \in \boldsymbol{N}$, $h_{1}, h_{2}, \ldots, h_{n} \in \boldsymbol{H}$ and a real polynomial $p\left(x_{1}, x_{2}, \ldots, x_{n}\right)$ of $n$-variables such that $f(w)=p\left(\left[h_{1}\right](w),\left[h_{2}\right](w), \ldots,\left[h_{n}\right](w)\right)$ for $h_{i}=\left(h_{i}^{j}\right) \in \boldsymbol{H}$, where

$$
\left[h_{i}\right](w)=\sum_{j=1}^{m} \int_{0}^{T} \dot{h}_{i}^{j} d w^{j} .
$$

are defined in the sense of Itô's stochastic integrals.

The standard $L_{p}$-norm of the $\boldsymbol{R}$-valued Wiener functional $F$ is defined by $\|F\|_{p}=\left(\int_{W}|F|^{p} P(d \omega)\right)^{1 / p}$. Also a sequence of the norms of the $\boldsymbol{R}$-valued Wiener functional $F$ for any $s \in \boldsymbol{R}$, and $p \in(1, \infty)$ is defined by

$$
\|F\|_{p, s}=\left\|(I-\mathcal{L})^{s / 2} F\right\|_{p},
$$

where $\mathcal{L}$ is the Ornstein-Uhlenbeck operator and $\|\cdot\|_{p}$ is the $L_{p}$-norm in the stochastic analysis. The O-U operator in (2.3) means that $(I-\mathcal{L})^{s / 2} F=$ $\sum_{n=0}^{\infty}(1+n)^{s / 2} J_{n} F$, where $J_{n}$ are the projection operators in the Wiener 
homogeneous chaos decomposition in $L_{2}(\boldsymbol{R})$. They are constructed by the totality of $\boldsymbol{R}$-valued polynomials of degree at most $n$, denoted by $\boldsymbol{P}_{n}$.

Let $\boldsymbol{P}(\boldsymbol{R})$ denote the totality of $\boldsymbol{R}$-valued polynomials on the Wiener space $(\boldsymbol{W}, P)$. Then $\boldsymbol{P}(\boldsymbol{R})$ is dense in $L_{p}(\boldsymbol{R})$ and can be extended to the totality of smooth functionals $S$ (the $C^{\infty}$ functions with derivatives of polynomial growth orders). Then we can construct the Banach space $\boldsymbol{D}_{p}^{s}(\boldsymbol{R})$ as the completion of $\boldsymbol{P}(\boldsymbol{R})$ with respect to $\|\cdot\|_{p, s}$. The dual space of $\boldsymbol{D}_{p}^{s}(\boldsymbol{R})$ is $\boldsymbol{D}_{q}^{-s}(\boldsymbol{R})$, where $s \in \boldsymbol{R}$, $p>1$ and $1 / p+1 / q=1$. The space $\boldsymbol{D}^{\infty}(\boldsymbol{R})=\bigcap_{s>0} \bigcap_{1<p<+\infty} \boldsymbol{D}_{p}^{s}(\boldsymbol{R})$ is the set of Wiener functionals and $\tilde{\boldsymbol{D}}^{-\infty}(\boldsymbol{R})=\bigcup_{s>0} \bigcap_{1<p<+\infty} \boldsymbol{D}_{p}^{-s}(\boldsymbol{R})$ is a space of generalized Wiener functionals. For $F \in \boldsymbol{P}(\boldsymbol{R})$ and $h \in \boldsymbol{H}$, the derivative of $F$ in the direction of $h$ is defined by

$$
D_{h} F(w)=\lim _{\varepsilon \rightarrow 0} \frac{1}{\varepsilon}\{F(w+\varepsilon h)-F(w)\} .
$$

Then for $F \in \boldsymbol{P}(\boldsymbol{R})$ and $h \in \boldsymbol{H}$ there exists $D F \in \boldsymbol{P}(\boldsymbol{H} \otimes \boldsymbol{R})$ such that $D_{h} F(w)=$ $\langle D F(w), h\rangle_{H}$, where $\langle\cdot\rangle_{H}$ is the inner product of $\boldsymbol{H}$ and $D F$ is called the $H$-derivative of $F$. Also for $F \in S(R)$ there exists a unique $D F \in S(H \otimes R)$.

More generally, for a separable Hilbert space $\boldsymbol{E}$, a function $f: \boldsymbol{W} \mapsto \boldsymbol{E}$ is called a polynomial functional if there exist $n \in \boldsymbol{N}, h_{1}, h_{2}, \ldots, h_{n} \in \boldsymbol{H}$ and real polynomials $p_{i}\left(x_{1}, x_{2}, \ldots, x_{n}\right)$ of $n$-variables such that

$$
f(w)=\sum_{i=1}^{d} p_{i}\left(\left[h_{1}\right](w),\left[h_{2}\right](w), \ldots,\left[h_{n}\right](w)\right) e_{i}
$$

for some $d \in \boldsymbol{N}$, where $e_{1}, \ldots, e_{d} \in \boldsymbol{E}$. The totality of $\boldsymbol{E}$-valued polynomial functions and the totality of $\boldsymbol{E}$-valued smooth functionals are denoted by $\boldsymbol{P}(\boldsymbol{E})$ and $\boldsymbol{S}(\boldsymbol{E})$, respectively. By extending the above construction for $\boldsymbol{P}(\boldsymbol{R})$ to $\boldsymbol{S}(\boldsymbol{E})$, there exists $D F \in \boldsymbol{S}(\boldsymbol{H} \otimes \boldsymbol{E})$ such that $D_{h} F(w)=\langle D F(w), h\rangle_{H}$, where $\langle\cdot\rangle_{H}$ is the inner product of $\boldsymbol{H}$.

By repeating this procedure, we can sequentially define the $k$ th order $H$-derivative $D^{k} F \in \boldsymbol{S}\left(\boldsymbol{H}^{\otimes k} \otimes \boldsymbol{E}\right)$ for $k \geq 1$, and it is known that the norm $\|\cdot\|_{p, s}$ is equivalent to the norm $\sum_{k=0}^{s}\left\|D^{k} \cdot\right\|_{p}$. In particular, for $F=\left(F^{i}\right) \in \boldsymbol{D}_{p}^{1}\left(\boldsymbol{R}^{d}\right)$, we define the Malliavin covariance by

$$
\sigma_{\mathrm{MC}}(F)=\left(\left\langle D F^{i}(w), D F^{j}(w)\right\rangle_{H}\right), \quad i, j=1, \ldots, d .
$$

2.2. Asymptotic expansions. Let $X^{(\varepsilon)}(w)=\left(X^{i(\varepsilon)}(w)\right)(i=1, \ldots, d ; \varepsilon \in$ $(0,1])$ be a Wiener functional with a parameter $\varepsilon$. Then we need to define the asymptotic expansion of $X^{(\varepsilon)}(w)$ with respect to $\varepsilon$ in the proper mathematical sense. For $k>0, X^{(\varepsilon)}(w)=O\left(\varepsilon^{k}\right)$ in $\boldsymbol{D}_{p}^{s}(\boldsymbol{E})$ as $\varepsilon \downarrow 0$ means that

$$
\lim \sup _{\varepsilon \downarrow 0} \frac{\left\|X^{(\varepsilon)}\right\|_{p, s}}{\varepsilon^{k}}<+\infty .
$$


If for all $p>1, s>0$ and every $k=1,2, \ldots$,

$$
X^{(\varepsilon)}(w)-\left(g_{1}+\varepsilon g_{2}+\cdots+\varepsilon^{k-1} g_{k}\right)=O\left(\varepsilon^{k}\right)
$$

in $\boldsymbol{D}_{p}^{s}(\boldsymbol{E})$ as $\varepsilon \downarrow 0$, then we say that $X^{(\varepsilon)}(w)$ has an asymptotic expansion

$$
X^{(\varepsilon)}(w) \sim g_{1}+\varepsilon g_{2}+\cdots
$$

in $\boldsymbol{D}^{\infty}(\boldsymbol{E})$ as $\varepsilon \downarrow 0$ with $g_{1}, g_{2}, \ldots \in \boldsymbol{D}^{\infty}(\boldsymbol{E})$.

Also if for every $k=1,2, \ldots$, there exists $s>0$ such that, for all $p>1$, $X^{(\varepsilon)}(w), g_{1}, g_{2}, \ldots \in \boldsymbol{D}_{p}^{-s}(\boldsymbol{E})$ and

$$
X^{(\varepsilon)}(w)-\left(g_{1}+\varepsilon g_{2}+\cdots+\varepsilon^{k-1} g_{k}\right)=O\left(\varepsilon^{k}\right)
$$

in $\boldsymbol{D}_{p}^{-s}(\boldsymbol{E})$ as $\varepsilon \downarrow 0$, then we say that $X^{(\varepsilon)}(w) \in \tilde{\boldsymbol{D}}^{-\infty}(\boldsymbol{E})$ has an asymptotic expansion

$$
X^{(\varepsilon)}(w) \sim g_{1}+\varepsilon g_{2}+\cdots
$$

in $\tilde{\boldsymbol{D}}^{-\infty}(\boldsymbol{E})$ as $\varepsilon \downarrow 0$ with $g_{1}, g_{2}, \ldots \in \tilde{\boldsymbol{D}}^{-\infty}(\boldsymbol{E})$.

Let $\boldsymbol{s}\left(\boldsymbol{R}^{d}\right)$ be the totality of $C^{\infty}$ rapidly decreasing functions on $\boldsymbol{R}^{d}$ and $\boldsymbol{\delta}^{\prime}\left(\boldsymbol{R}^{d}\right)$ be its dual. Also let $\eta^{\varepsilon} \in \boldsymbol{D}^{\infty}(\boldsymbol{R})$ and $\psi(y)$ be a smooth function such that $0 \leq \psi(y) \leq 1$ for $y \in \boldsymbol{R}, \psi(y)=1$ for $|y| \leq 1 / 2$ and $\psi=0$ for $|y| \geq 1$. It is known that if for any $p>1$ the Malliavin covariance of $X^{(\varepsilon)} \in \boldsymbol{D}^{\infty}\left(\boldsymbol{R}^{d}\right)$ satisfies

$$
\sup _{\varepsilon \in(0,1]} \mathbf{E}\left[\mathbb{1}_{\left\{\left|\eta^{\varepsilon}\right| \leq 1\right\}}\left(\operatorname{det}\left[\sigma_{\mathrm{MC}}\left(X^{(\varepsilon)}\right)\right]\right)^{-p}\right]<\infty,
$$

the composite functional $\hat{G}=\psi\left(\eta^{\varepsilon}\right) G \circ X^{(\varepsilon)} \in \tilde{\boldsymbol{D}}^{-\infty}\left(\boldsymbol{R}^{d}\right)$ is well defined with any $G \in \boldsymbol{S}^{\prime}\left(\boldsymbol{R}^{d}\right)$. Then the coupling

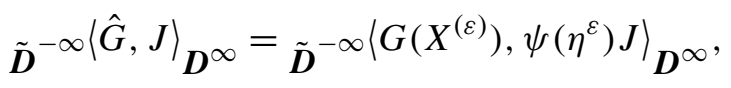

for any $J \in \boldsymbol{D}^{\infty}\left(\boldsymbol{R}^{d}\right)$, is well defined and we can use the notation of the expectation $\mathbf{E}\left[\psi\left(\eta^{\varepsilon}\right) G\left(X^{(\varepsilon)}\right)\right]$ by taking $J=\mathbf{1}$. With these formulations and notation we are ready to state a simplified version of Theorem 2.2 of Yoshida (1992), which is a truncated version of Theorem 2.3 of Watanabe (1987). The validity of the asymptotic expansion is obtained by showing that the conditions of the next theorem are met in our situations.

THEOREM 2.1 [Yoshida (1992)]. Suppose the following set of sufficient conditions are satisfied:

(i) $\left\{X^{(\varepsilon)}(w) ; \varepsilon \in(0,1]\right\} \in D^{\infty}\left(\boldsymbol{R}^{d}\right)$ with $X^{(\varepsilon)}(w)=\left(X^{i(\varepsilon)}(w)\right)$.

(ii) $X^{(\varepsilon)}(w)$ has the asymptotic expansion $X^{(\varepsilon)}(w) \sim g_{1}+\varepsilon g_{2}+\cdots$ in $\boldsymbol{D}^{\infty}\left(\boldsymbol{R}^{d}\right)$ as $\varepsilon \downarrow 0$ with $g_{1}, g_{2}, \ldots \in \boldsymbol{D}^{\infty}\left(\boldsymbol{R}^{d}\right)$.

(iii) $\left\{\eta^{\varepsilon}(w) ; \varepsilon \in(0,1]\right\} \subset \boldsymbol{D}^{\infty}(\boldsymbol{R})$ and it is $O(1)$ in $\boldsymbol{D}^{\infty}(\boldsymbol{R})$ as $\varepsilon \downarrow 0$. 
(iv) For any $p>1$,

$$
\sup _{\varepsilon \in(0,1]} \mathbf{E}\left[\mathbb{1}_{\left\{\left|\eta^{\varepsilon}\right| \leq 1\right\}}\left(\operatorname{det}\left[\sigma_{\mathrm{MC}}\left(X^{(\varepsilon)}\right)\right]\right)^{-p}\right]<\infty .
$$

(v) For any $k \geq 1$,

$$
\lim _{\varepsilon \rightarrow 0} \varepsilon^{-k} P\left\{\left|\eta^{\varepsilon}\right|>\frac{1}{2}\right\}=0
$$

(vi) Let $\phi^{(\varepsilon)}(x)$ be a smooth function in $(x, \varepsilon)$ on $\boldsymbol{R}^{d} \times(0,1]$ with all derivatives of polynomial growth order in $x$ uniformly in $\varepsilon$.

Then $\psi\left(\eta^{\varepsilon}\right) \phi^{(\varepsilon)}\left(X^{(\varepsilon)}\right) I_{\mathcal{B}}\left(X^{(\varepsilon)}\right)$ has an asymptotic expansion,

$$
\psi\left(\eta^{\varepsilon}\right) \phi^{(\varepsilon)}\left(X^{(\varepsilon)}\right) I_{B}\left(X^{(\varepsilon)}\right) \sim \Phi_{0}+\varepsilon \Phi_{1}+\cdots
$$

in $\tilde{\boldsymbol{D}}^{-\infty}(\boldsymbol{R})$ as $\varepsilon \downarrow 0$, where $I_{\mathcal{B}}$ is the indicator function for any Borel set $\mathcal{B}$ and $\Phi_{0}, \Phi_{1}, \ldots$ are determined by the formal Taylor expansion with respect to $X^{(\varepsilon)}$ in (2.10).

REMARK. We have to mention an intuitive meaning of the asymptotic expansion in the above theorem. If we truncate the random variable under the condition of (2.13), then the asymptotic expansion in (2.15) implies

$$
\begin{aligned}
\underset{\varepsilon \downarrow 0}{\limsup } \mid \frac{1}{\varepsilon^{k}} \mathbf{E}\left[\psi\left(\eta_{c}^{\varepsilon}\right) \phi^{(\varepsilon)}\left(X^{(\varepsilon)}\right) I_{\mathcal{B}}\left(X^{(\varepsilon)}\right)\right. \\
\left.-\left(\Phi_{0}+\varepsilon \Phi_{1}+\cdots+\varepsilon^{k-1} \Phi_{k-1}\right)\right] \mid<+\infty
\end{aligned}
$$

for any integer $k \geq 1$ if we use the expectation operation in the proper mathematical sense. The calculations of the generalized expectation operations for the generalized Wiener functionals will be discussed in Section 3.

3. Validity in the Black-Scholes economy. Let $\left(\Omega, \mathcal{F}, Q,\left\{\mathcal{F}_{t}\right\}_{t \in[0, T]}\right)$ be the filtered probability space with $T<+\infty$. For $\varepsilon \in(0,1]$ and $0<t \leq T$, the vector of $d$ security prices follow a sequence of stochastic differential equations

$$
S_{t}^{(\varepsilon)}=S_{0}+\int_{0}^{t} \mu\left(S_{s}^{(\varepsilon)}, s\right) d s+\int_{0}^{t} \varepsilon \sigma\left(S_{s}^{(\varepsilon)}, s\right) d w_{s},
$$

where $\mu\left(S_{s}^{(\varepsilon)}, s\right)=r\left(S_{s}^{(\varepsilon)}, s\right) S_{s}^{(\varepsilon)}$ and $\sigma\left(S_{s}^{(\varepsilon)}, s\right)=\left(\sigma^{i j}\left(S_{s}^{(\varepsilon)}, s\right)\right)$ are $R^{d} \times$ $[0, T] \rightarrow R^{d}$ and $R^{d} \times[0, T] \rightarrow R^{d} \otimes R^{m}$ Borel measurable functions in $\left(S_{s}^{(\varepsilon)}, s\right)$, respectively, and $w_{s}\left[=\left(w_{s}^{i}\right)\right]$ are the vector of $m \times 1$ Brownian motions with respect to $\left\{\mathcal{F}_{t}\right\}$. We further assume that the drift and the volatility functions are continuous and $\boldsymbol{C}^{\infty}$ for $s \in[0, T]$ with bounded derivatives of any order in the first 
argument. That is, for the first argument there exist positive constants $M_{1}(k)$ and $M_{2}(k)(k \geq 1)$ such that for any $i=1, \ldots, d$ and $j=1, \ldots, m$,

$$
\begin{aligned}
& \sup _{S \in R^{d}, 0 \leq s \leq T}\left|\frac{\partial^{k} \mu^{i}\left(S_{S}^{(\varepsilon)}, s\right)}{\partial S_{S}^{i_{1}(\varepsilon)} \cdots \partial S_{S}^{i_{k}(\varepsilon)}}\right|<M_{1}(k), \\
& \sup _{S \in R^{d}, 0 \leq s \leq T}\left|\frac{\partial^{k} \sigma^{i j}\left(S_{S}^{(\varepsilon)}, s\right)}{\partial S_{S}^{i_{1}(\varepsilon)} \cdots \partial S_{S}^{i_{k}(\varepsilon)}}\right|<M_{2}(k),
\end{aligned}
$$

where $\mu\left(S_{S}^{(\varepsilon)}, s\right)=\left(\mu^{i}\left(S_{s}^{(\varepsilon)}, s\right)\right)$, and we shall denote the partial derivatives as $\partial_{i_{1}, \ldots, i_{k}}^{k} \mu^{i}\left(S_{s}^{(\varepsilon)}, s\right)$ and $\partial_{i_{1}, \ldots, i_{k}}^{k} \sigma^{i j}\left(S_{s}^{(\varepsilon)}, s\right)$, respectively. We further consider the case that there exists a positve $M_{3}$ such that

$$
\sup _{0 \leq s \leq T}[|\mu(0, s)|+|\sigma(0, s)|]<M_{3},
$$

where the notation $|A|=\sqrt{\sum_{i, j}\left|a^{i j}\right|^{2}}$ for any matrix $A=\left(a^{i j}\right)$ and $|a|=$ $\sqrt{\sum_{i}\left|a^{i}\right|^{2}}$ for any vector $a=\left(a^{i}\right)$ are used. These conditions imply that there exists some positive $K_{i}>0(i=1,2)$ such that for all $s, t \in[0, T]$,

$$
\begin{array}{r}
\left|\mu\left(S_{s}^{(\varepsilon)}, s\right)\right|+\left|\sigma\left(S_{s}^{(\varepsilon)}, s\right)\right|<K_{1}\left(1+\left|S_{s}^{(\varepsilon)}\right|\right), \\
\left|\mu\left(S_{1 s}^{(\varepsilon)}, s\right)-\mu\left(S_{2 s}^{(\varepsilon)}, s\right)\right|+\left|\sigma\left(S_{1 s}^{(\varepsilon)}, s\right)-\sigma\left(S_{2 s}^{(\varepsilon)}, s\right)\right|<K_{2}\left|S_{1 s}^{(\varepsilon)}-S_{2 s}^{(\varepsilon)}\right| .
\end{array}
$$

Then the standard argument in stochastic analysis shows the existence of the unique strong solution which has continuous sample paths and is in $L_{p}\left(\boldsymbol{R}^{d}\right)$ for any $1<p<\infty$. In the remainder of this section, we will mainly discuss the validity of the asymptotic expansion of $\phi\left(X_{T}^{(\varepsilon)}\right) I_{\mathcal{B}}\left(X_{T}^{(\varepsilon)}\right)$ for any Borel set $\mathcal{B}$, where $X_{T}^{(\varepsilon)}$ is defined by

$$
X_{T}^{(\varepsilon)}=\frac{1}{\varepsilon}\left(S_{T}^{(\varepsilon)}-S_{T}^{(0)}\right)
$$

and $S_{T}^{(0)}$ is the solution of the ordinary differential equation

$$
S_{T}^{(0)}=S_{0}+\int_{0}^{T} \mu\left(S_{s}^{(0)}, s\right) d s .
$$

For illustrations in this section, we only mention simple examples. When we take $d=m=1, \phi(x)=(x+y)$ and $I_{\mathcal{B}}(x)=\{x \geq-y\}$ for a constant $y$, it corresponds to the valuation problem of European options in mathematical finance. We shall give another example for the Asian options, which was considered by Kunitomo and Takahashi (1992).

First, we shall show that $S_{T}^{(\varepsilon)}$ is a smooth Wiener functional in the sense of Malliavin. A more detailed proof when $d=m=1$ has been discussed by Kunitomo and Takahashi (1998) and Takahashi (1999). 
THEOREM 3.1. Under the assumptions in (3.1)-(3.3), $S_{T}^{(\varepsilon)}$ is in $\boldsymbol{D}^{\infty}\left(\boldsymbol{R}^{d}\right)$ and has an asymptotic expansion

$$
S_{T}^{(\varepsilon)} \sim S_{T}^{(0)}+\varepsilon g_{1 T}+\varepsilon^{2} g_{2 T}+\cdots
$$

as $\varepsilon \downarrow 0$ with $g_{1 T}, g_{2 T}, \ldots \in \boldsymbol{D}^{\infty}\left(\boldsymbol{R}^{d}\right)$.

Proof. (i) The first part of our proof is to show that $S_{T}^{(\varepsilon)}$ is in $\boldsymbol{D}^{\infty}\left(\boldsymbol{R}^{d}\right)$. But it is well known that $S_{T}^{(\varepsilon)} \in \boldsymbol{D}^{\infty}\left(\boldsymbol{R}^{d}\right)$ when $S_{T}^{(\varepsilon)}$ follows a time-homogeneous Markovian process. Since any time-dependent Markovian process can be represented as a time-homogeneous Markovian process, we can immediately apply the general result to our case. [See Chapter V of Ikeda and Watanabe (1989), or Kusuoka and Strook (1982).]

(ii) We shall prove the second part of Theorem 3.1. The coefficients appearing in the asymptotic expansion of $S_{T}^{(\varepsilon)}$ are given by the formal Taylor formula. By expanding $X_{T}^{(\varepsilon)}$ as $X_{T}^{(\varepsilon)}=g_{1 T}+\varepsilon g_{2 T}+\varepsilon^{2} g_{3 T}+\cdots$ with respect to $\varepsilon$, we can determine the coefficients $\left\{g_{j T}(j \geq 1)\right\}$ recursively. The $i$ th component of the leading term $(i=1,2, \ldots, d)$ is given by

$$
g_{1 T}^{(i)}=\sum_{l_{1}=1}^{d} \int_{0}^{T} \partial_{l_{1}} \mu^{i}\left(S_{s}^{(0)}, s\right) g_{1 s}^{\left(l_{1}\right)} d s+\sum_{j^{\prime}=1}^{m} \int_{0}^{T} \sigma^{i j^{\prime}}\left(S_{s}^{(0)}, s\right) d w_{s}^{j^{\prime}} .
$$

Then it can be written as

$$
g_{1 T}^{(i)}=\sum_{j=1}^{d} \sum_{j^{\prime}=1}^{m} \int_{0}^{T}\left(Y_{T} Y_{s}^{-1}\right)^{i j} \sigma^{j j^{\prime}}\left(S_{s}^{(0)}, s\right) d w_{s}^{j^{\prime}},
$$

where $Y_{t}=Y_{t}^{(0)}$ is the solution of the ordinary differential equation $d Y^{i l}=$ $\sum_{k=1}^{d} \partial_{k} \mu^{i}\left(S_{t}^{(0)}, t\right) Y^{k l} d t$. This equation can be solved and its solution is written as $Y_{t}=\exp \left(\int_{0}^{t}\left(\partial_{j} \mu^{i}\left(S_{s}^{(0)}, s\right)\right) d s\right)$ with the initial condition $Y_{0}=I_{d}$.

For $n \geq 2$, we recursively define the $i$ th component of each term $g_{n T}^{(i)}$ by

$$
\begin{aligned}
g_{n T}^{(i)}= & \sum_{\substack{k=1 \\
m_{1}+\cdots+m_{k}=n}}^{n} \int_{0}^{T}\left[\frac{1}{k !} \sum_{l_{1}, \ldots, l_{k}=1}^{d} \partial_{l_{1}, \ldots, l_{k}}^{k} \mu^{i}\left(S_{s}^{(0)}, s\right) \prod_{j=1}^{k} g_{m_{j}, s}^{\left(l_{j}\right)}\right] d s \\
& +\sum_{\substack{k=1 \\
m_{1}+\cdots+m_{k}=n-1}}^{n} \int_{0}^{T}\left[\frac{1}{k !} \sum_{l_{1}, \ldots, l_{k}=1}^{d} \sum_{j^{\prime}=1}^{m} \partial_{l_{1}, \ldots, l_{k}}^{k} \sigma^{i j^{\prime}}\left(S_{s}^{(0)}, s\right) \prod_{j=1}^{k} g_{m_{j}, s}^{\left(l_{j}\right)} d w_{s}^{j^{\prime}}\right],
\end{aligned}
$$

where $m_{j}(j=1, \ldots, k)$ are positive integers.

By the boundedness of $Y_{T}, Y_{s}^{-1}, \sigma\left(S_{s}^{(0)}, s\right)$ on $[0, T]$, we have $\mathbf{E}\left[\left|g_{1 s}\right|^{p}\right]<\infty$, $s \in[0, T]$ for any $1<p<\infty$. Given $g_{1 s} \in L_{p}\left(\boldsymbol{R}^{d}\right)$, we have $\mathbf{E}\left[\left|g_{2 s}\right|^{p}\right]<\infty$ for 
any $1<p<\infty$. By the same token, the relation $g_{k s} \in L_{p}\left(\boldsymbol{R}^{d}\right)$ can be obtainable recursively given $g_{j s} \in L_{p}\left(\boldsymbol{R}^{d}\right)(j=1,2, \ldots k-1)$ and we have $g_{1 T}, g_{2 T}, \ldots \in$ $\bigcap_{1<p<\infty} \boldsymbol{D}_{p}^{1}\left(\boldsymbol{R}^{d}\right)$.

Next, we note that

$$
D_{h} g_{1 T}^{(i)}=\sum_{j=1}^{d} \sum_{j^{\prime}=1}^{m} \int_{0}^{T}\left(Y_{T} Y_{s}^{-1}\right)^{i j} \sigma^{j j^{\prime}}\left(S_{s}^{(0)}, s\right) \dot{h}_{s}^{j^{\prime}} d s .
$$

For higher order derivatives we use an induction argument and we assume $g_{n T} \in \bigcap_{1<p<\infty} \boldsymbol{D}_{p}^{k}\left(\boldsymbol{R}^{d}\right)(k \geq 1)$ for any $n \geq 1$. Then we need to show that $g_{n T} \in \bigcap_{1<p<\infty} \boldsymbol{D}_{p}^{k+1}\left(\boldsymbol{R}^{d}\right)$. Actually, we can show the $L_{p}$-boundedness of any order $H$-derivatives of $g_{n T}(n \geq 1)$ recursively. In our evaluations of higher order $H$-derivatives, we need a version of Burkholder's inequality for Hilbert space valued stochastic integrals proved by Lemma 2.1 of Kusuoka and Strook (1982). Given $g_{1 s} \in \boldsymbol{D}^{\infty}\left(\boldsymbol{R}^{d}\right)$ for any $s \in[0, T]$, we can recursively show that $g_{n T} \in$ $\boldsymbol{D}^{\infty}\left(\boldsymbol{R}^{d}\right)$.

(iii) Finally, for any $n(n \geq 1)$ and $s \in[0, T]$ let

$$
Z_{n s}^{(\varepsilon)}=\frac{1}{\varepsilon^{n}}\left[X_{s}^{(\varepsilon)}-g_{1 s}-\varepsilon g_{2 s}-\cdots-\varepsilon^{n-1} g_{n s}\right] .
$$

By using (3.1) repeatedly and applying the standard arguments, we can show that $Z_{1 s}^{(\varepsilon)} \in L_{p}\left(\boldsymbol{R}^{d}\right)$ for any $p>1$ uniformly with respect to $\varepsilon$. Again, by applying recursive arguments and using induction with respect to $n$, we can show that $Z_{n s}^{(\varepsilon)}$ and their $H$-derivatives are in $L_{p}$ uniformly with respect to $\varepsilon$ after tedious arguments, which were omitted.

We now return to the original problem on the normalized random variable $X_{T}^{(\varepsilon)}$ in (3.7). Using Theorem 3.1, we see $X_{T}^{(\varepsilon)}$ is in $\boldsymbol{D}^{\infty}\left(\boldsymbol{R}^{d}\right)$ and has a proper asymptotic expansion

$$
X_{T}^{(\varepsilon)} \sim g_{1 T}+\varepsilon g_{2 T}+\cdots
$$

in $\boldsymbol{D}^{\infty}\left(\boldsymbol{R}^{d}\right)$ with $g_{1 T}, g_{2 T}, \ldots \in \boldsymbol{D}^{\infty}\left(\boldsymbol{R}^{d}\right)$. By using the Fubini-type result in our setting for any $h \in \boldsymbol{H}$, the first-order $H$-derivative $D_{h} S_{T}^{(\varepsilon)}$ satisfies

$$
\begin{aligned}
D_{h} S_{T}^{(\varepsilon) i}= & \sum_{j=1}^{m} \sum_{k=1}^{d} \int_{0}^{T} \varepsilon \partial_{k} \sigma^{i j}\left(S_{s}^{(\varepsilon)}, s\right) D_{h} S_{s}^{(\varepsilon) k} d w^{j}(s) \\
& +\sum_{k=1}^{d} \int_{0}^{T} \partial_{k} \mu^{i}\left(S_{s}^{(\varepsilon)}, s\right) D_{h} S_{s}^{(\varepsilon) k} d s+\sum_{j=1}^{m} \int_{0}^{T} \varepsilon \sigma^{i j}\left(S_{s}^{(\varepsilon)}, s\right) \dot{h}_{s}^{j} d s .
\end{aligned}
$$

Then it can be represented as

$$
D_{h} S_{T}^{(\varepsilon) i}=\sum_{j=1}^{d} \sum_{j^{\prime}=1}^{m} \int_{0}^{T}\left(Y_{T}^{(\varepsilon)} Y_{s}^{(\varepsilon)-1}\right)^{i j} \varepsilon \sigma^{j j^{\prime}}\left(S_{s}^{(\varepsilon)}, s\right) \dot{h}_{s}^{j^{\prime}} d s
$$


where $Y_{t}^{(\varepsilon)}$ is the solution of the stochastic differential equation

$$
d Y_{t}^{(\varepsilon) i l}=\sum_{k=1}^{d} \partial_{k} \mu^{i}\left(S_{t}^{(\varepsilon)}, t\right) Y_{t}^{(\varepsilon) k l} d t+\varepsilon \sum_{j=1}^{m} \sum_{k=1}^{d} \partial_{k} \sigma^{i j}\left(S_{t}^{(\varepsilon)}, t\right) Y_{t}^{(\varepsilon) k l} d w_{t}^{j}
$$

and $Y_{0}^{(\varepsilon)}=I_{d}$. Then the Malliavin covariance of the normalized random variable $\sigma_{\mathrm{MC}}\left(X_{T}^{(\varepsilon)}\right)=\left(\sigma_{\mathrm{MC}}^{i j}\left(X_{T}^{(\varepsilon)}\right)\right)$ is given by

$$
\sigma_{\mathrm{MC}}^{i j}\left(X_{T}^{(\varepsilon)}\right)=\sum_{k=1}^{m} \int_{0}^{T}\left[Y_{T}^{(\varepsilon)} Y_{s}^{(\varepsilon)-1} \sigma\left(S_{s}^{(\varepsilon)}, s\right)\right]^{i k}\left[Y_{T}^{(\varepsilon)} Y_{s}^{(\varepsilon)-1} \sigma\left(S_{s}^{(\varepsilon)}, s\right)\right]^{j k} d s .
$$

We shall consider the uniform nondegeneracy of the Malliavin covariance, which is the most important step in the application of Theorem 2.1. For this purpose, we need the following assumption.

ASSUMPTION I. For any $T>0$ the $d \times d$ matrix $\Sigma_{g_{1}}=\left(\Sigma_{g_{1}}^{i j}\right)$ is positive definite, where $\Sigma_{g_{1}}^{i j}$ is given by

$$
\Sigma_{g_{1}}^{i j}=\sum_{k=1}^{m} \int_{0}^{T}\left[Y_{T} Y_{s}^{-1} \sigma\left(S_{s}^{(0)}, s\right)\right]^{i k}\left[Y_{T} Y_{s}^{-1} \sigma\left(S_{s}^{(0)}, s\right)\right]^{j k} d s .
$$

This assumption assures the nondegeneracy of the limiting distribution of the random variable, which can be easily checked in applications. We define $\eta_{c}^{\varepsilon}$ as

$$
\eta_{c}^{\varepsilon}=c \int_{0}^{T}\left|Y_{T}^{(\varepsilon)}\left(Y_{s}^{(\varepsilon)}\right)^{-1} \sigma\left(S_{s}^{(\varepsilon)}, s\right)-Y_{T} Y_{s}^{-1} \sigma\left(S_{s}^{(0)}, s\right)\right|^{2} d s
$$

for any $c>0$. Let $\xi_{s, t}^{(\varepsilon)}=Y_{t}^{(\varepsilon)}\left(Y_{s}^{\varepsilon}\right)^{-1} \sigma\left(S_{s}^{(\varepsilon)}, s\right)$ and $\xi_{s, t}=Y_{t} Y_{s}^{-1} \sigma\left(S_{s}^{(0)}, s\right)$ and we note an inequality $\left|\xi_{s, T}^{(\varepsilon)} \xi_{s, T}^{(\varepsilon) *}-\xi_{s, T} \xi_{s, T}^{*}\right| \leq\left|\xi_{s, T}^{(\varepsilon)}-\xi_{s, T}\right|^{2}+2\left|\xi_{s, T}\right|\left|\xi_{s, T}^{(\varepsilon)}-\xi_{s, T}\right|$, where the notation $A^{*}$ is used for the transpose of any matrix $A=\left(a_{i j}\right)$. Then condition $\left|\eta_{c}^{\delta}\right| \leq 1$ is equivalent to $\int_{0}^{T}\left|\xi_{s, T}^{(\varepsilon)}-\xi_{s, T}\right|^{2} d s \leq 1 / c$ and we have

$$
\left|\sigma_{\mathrm{MC}}\left(X_{T}^{(\varepsilon)}\right)-\Sigma_{g_{1}}\right| \leq \frac{1}{c}+2\left|\Sigma_{g_{1}}\right| \sqrt{\frac{1}{c}}
$$

for $\left|\eta_{c}^{\varepsilon}\right| \leq 1$. Thus we can take $c_{0}$ such that for any $c>c_{0}>0,0<\Sigma_{g_{1}}+$ $\left(\sigma_{\mathrm{MC}}\left(X_{T}^{(\varepsilon)}\right)-\Sigma_{g_{1}}\right)=\sigma_{\mathrm{MC}}\left(X_{T}^{(\varepsilon)}\right)$ holds uniformly for $\varepsilon \in(0,1]$. Hence we have the next result on the uniform nondegeneracy of the Malliavin covariance.

THEOREM 3.2. Under the assumptions in (3.1)-(3.3) and Assumption I, the Malliavin covariance $\sigma_{\mathrm{MC}}\left(X_{T}^{(\varepsilon)}\right)$ is uniformly nondegenerate. That is, there exists $c_{0}>0$ such that for $c>c_{0}$ and any $p>1$,

$$
\sup _{\varepsilon \in(0,1]} \mathbf{E}\left[\mathbb{1}_{\left\{\eta_{c}^{\varepsilon} \leq 1\right\}}\left\{\operatorname{det}\left(\sigma_{\mathrm{MC}}\left(X_{T}^{(\varepsilon)}\right)\right)\right\}^{-p}\right]<\infty .
$$


By using the results in Theorem 3.1, Theorem 3.2 and Lemma A.2 in the Appendix, we have shown that the conditions of Theorem 2.1 are satisfied. Then we immediately obtain the next result.

THEOREM 3.3. Under the assumptions in (3.1)-(3.3) and Assumption I, for a smooth function $\phi^{(\varepsilon)}(x)$ with all derivatives of polynomial growth orders, $\psi\left(\eta_{c}^{\varepsilon}\right) \phi^{(\varepsilon)}\left(X_{T}^{(\varepsilon)}\right) I_{\mathcal{B}}\left(X_{T}^{(\varepsilon)}\right)$ has an asymptotic expansion

$$
\psi\left(\eta_{c}^{\varepsilon}\right) \phi^{(\varepsilon)}\left(X_{T}^{(\varepsilon)}\right) I_{\mathcal{B}}\left(X_{T}^{(\varepsilon)}\right) \sim \Phi_{0}+\varepsilon \Phi_{1}+\varepsilon^{2} \Phi_{2}+\cdots
$$

in $\tilde{\boldsymbol{D}}^{-\infty}(\boldsymbol{R})$ as $\varepsilon \downarrow 0$, where $\mathscr{B}$ is a Borel set, $\psi(x)$ is a smooth function such that $0 \leq \psi(x) \leq 1$ for $x \in R, \psi(x)=1$ for $|x| \leq 1 / 2, \psi=0$ for $|x| \geq 1$, and $\Phi_{0}, \Phi_{1}, \ldots$ are determined by the formal Taylor expansion with respect to $X_{T}^{(\varepsilon)}\left(\sim g_{1 T}+\varepsilon g_{2 T}+\cdots\right)$.

Then we obtain an asymptotic expansion of the expectation of (3.15), which is the direct consequence of Theorem 2.1 and Theorem 3.3.

COROLlaRY 3.1. Under the assumptions in (3.1)-(3.3) and Assumption I, an asymptotic expansion of $\mathbf{E}\left[\phi^{(\varepsilon)}\left(X_{T}^{(\varepsilon)}\right) I_{\mathcal{B}}\left(X_{T}^{(\varepsilon)}\right)\right]$ is given by

$$
\begin{aligned}
\mathbf{E}\left[\phi^{(\varepsilon)}\left(X_{T}^{(\varepsilon)}\right) I_{\mathcal{B}}\left(X_{T}^{(\varepsilon)}\right)\right] & \sim \mathbf{E}\left[\psi\left(\eta_{c}^{\varepsilon}\right) \phi^{(\varepsilon)}\left(X_{T}^{(\varepsilon)}\right) I_{\mathcal{B}}\left(X_{T}^{(\varepsilon)}\right)\right] \\
& \sim \mathbf{E}\left[\Phi_{0}\right]+\varepsilon \mathbf{E}\left[\Phi_{1}\right]+\varepsilon^{2} \mathbf{E}\left[\Phi_{2}\right]+\cdots
\end{aligned}
$$

as $\varepsilon \downarrow 0$, where $\phi^{(\varepsilon)}(\cdot), \psi(\cdot), \Phi_{j}(j \geq 0)$ and $\mathcal{B}$ are defined as in Theorem 3.3.

Our next objective is to show that the resulting formulas of asymptotic expansions are equivalent to those from the method based on the simple inversion technique for the characteristic function, which have been used by Kunitomo and Takahashi $(1995,2001)$, and Takahashi (1999). It is possible to explicitly derive the formulas of the asymptotic distribution function and the density function, and also those of the expectations of the random variables involving $X_{T}^{(\varepsilon)}$ in certain ranges. We start with the explicit evaluation of each terms appearing in Theorem 3.3. We observe that the first term in Theorem 3.3 is given by

$$
\Phi_{0}=\phi^{(0)}\left(g_{1 T}\right) I_{\mathcal{B}}\left(g_{1 T}\right) .
$$

Then by applying the Taylor expansion, for any $n \geq 1$, we have

$$
\begin{aligned}
\Phi_{n}=\sum_{\substack{k, l, m, k^{\prime}, m^{\prime} \geq 0 \\
k+l+m+k^{\prime}+m^{\prime}=n}}^{\substack{m_{1}+\cdots+m_{k}=m+2 k \\
m_{1}^{\prime}+\cdots+m_{k^{\prime}}^{\prime}=m^{\prime}+2 k^{\prime}}}\left(\sum_{l_{1}^{\prime}, \ldots, l_{k^{\prime}}^{\prime}=1}^{d} \frac{1}{k^{\prime} !} \partial_{l_{1}^{\prime}, \ldots, l_{k^{\prime}}}^{k^{\prime}} I_{\mathcal{B}}\left(g_{1 T}\right) \prod_{j=1}^{k^{\prime}} g_{m_{j}^{\prime} T}^{\left(l_{j^{\prime}}^{\prime}\right)}\right) \\
\times\left(\sum_{l_{1}, \ldots, l_{k}=1}^{d} \frac{1}{k ! l !} \partial_{l_{1}, \ldots, l_{k}}^{k} \partial_{\varepsilon}^{l} \phi^{(0)}\left(g_{1 T}\right) \prod_{j=1}^{k} g_{m_{j} T}^{\left(l_{j}\right)}\right),
\end{aligned}
$$


where $m_{j} \geq 2$ and $m_{j}^{\prime} \geq 2$.

When $d=m=1$ in particular, we have relatively simple and useful forms. For instance, the first two terms are given by

$$
\begin{aligned}
\Phi_{1}= & {\left[\frac{\partial \phi^{(0)}}{\partial \varepsilon}\left(g_{1 T}\right)+\partial \phi^{(0)}\left(g_{1 T}\right) g_{2}\right] I_{\mathcal{B}}\left(g_{1 T}\right)+\phi^{(0)}\left(g_{1 T}\right) \partial I_{\mathcal{B}}\left(g_{1 T}\right) g_{2 T}, } \\
\Phi_{2}= & {\left[\frac{\partial \phi^{(0)}}{\partial \varepsilon}\left(g_{1 T}\right)+\partial \phi^{(0)}\left(g_{1 T}\right) g_{2 T}\right] \partial I_{\mathcal{B}}\left(g_{1 T}\right) g_{2 T} } \\
& +\left[\frac{1}{2} \frac{\partial^{2} \phi^{(0)}}{\partial \varepsilon^{2}}\left(g_{1 T}\right)+\left\{\left.\frac{\partial^{2} \phi^{(0)}(x)}{\partial x \partial \varepsilon}\right|_{x=g_{1 T}}\right\} g_{2 T}\right. \\
& \left.\quad+\partial \phi^{(0)}\left(g_{1 T}\right) g_{3 T}+\frac{1}{2} \partial^{2} \phi^{(0)}\left(g_{1 T}\right) g_{2 T}^{2}\right] I_{\mathcal{B}}\left(g_{1 T}\right) \\
& +\phi^{(0)}\left(g_{1 T}\right)\left\{\frac{1}{2} \partial^{2} I_{\mathcal{B}}\left(g_{1 T}\right) g_{2 T}^{2}+\partial I_{\mathcal{B}}\left(g_{1 T}\right) g_{3 T}\right\} .
\end{aligned}
$$

In the above expressions the differentiations of the indicator function $I_{\mathcal{B}}(\cdot)$ have proper mathematical meanings as the generalized Wiener functionals. As we have indicated at the end of Section 2, the rigorous mathematical foundation of differentiation and the integration by parts formula have been given in Chapter $\mathrm{V}$ of Ikeda and Watanabe (1989) and Yoshida (1992, 1997). The next result summarizes the explicit expressions for the asymptotic expansion of expectations of the above random variables based on the Gaussian density function up to third-order terms.

THEOREM 3.4. In the asymptotic expansion of (3.16) terms $\mathbf{E}\left[\Phi_{n}\right](n=$ $0,1,2)$ are given by

$$
\begin{aligned}
\mathbf{E}\left[\Phi_{0}\right]= & \int_{\mathcal{B}} \phi^{(0)}(x) n\left[x \mid 0, \Sigma_{g_{1}}\right] d x, \\
\mathbf{E}\left[\Phi_{1}\right]=\int_{\mathcal{B}}\left\{\partial_{\varepsilon} \phi^{(0)}(x) n\left[x \mid 0, \Sigma_{g_{1}}\right]\right. & \\
& \left.\quad-\phi^{(0)}(x) \sum_{i=1}^{d} \partial_{i}\left\{\mathbf{E}\left[g_{2}^{(i)} \mid g_{1}=x\right] n\left[x \mid 0, \Sigma_{g_{1}}\right]\right\}\right\} d x, \\
\mathbf{E}\left[\Phi_{2}\right]= & \int_{\mathcal{B}}\left(-\partial_{\varepsilon} \phi^{(0)}(x) \sum_{i=1}^{d} \partial_{i}\left\{\mathbf{E}\left[g_{2}^{(i)} \mid g_{1}=x\right] n\left[x \mid 0, \Sigma_{g_{1}}\right]\right\}\right. \\
& +\frac{1}{2} \partial_{\varepsilon}^{2} \phi^{(0)}(x) n\left[x \mid 0, \Sigma_{g_{1}}\right]
\end{aligned}
$$




$$
\begin{aligned}
& -\frac{1}{2} \sum_{i, j=1}^{d} \phi^{(0)}(x) \partial_{i j}^{2}\left\{\mathbf{E}\left[g_{2}^{(i)} g_{2}^{(j)} \mid g_{1}=x\right] n\left[x \mid 0, \Sigma_{g_{1}}\right]\right\} \\
& \left.\quad-\sum_{i=1}^{d} \phi^{(0)}(x) \partial_{i}\left\{\mathbf{E}\left[g_{3}^{(i)} \mid g_{1}=x\right] n\left[x \mid 0, \Sigma_{g_{1}}\right]\right\}\right) d x
\end{aligned}
$$

where we denote $g_{n}^{(i)}=g_{n T}^{(i)}(i=1, \ldots, d ; n \geq 1), \partial_{\varepsilon} \phi^{(0)}=\left.\frac{\partial \phi^{(\varepsilon)}}{\partial \varepsilon}\right|_{\varepsilon=0}(x)$, $\mathbf{E}\left[z \mid g_{1}=x\right]$ as the conditional expectation of $z$ given $g_{1}=x$, and $n[x \mid 0, \Sigma]$ as the density function of the d-dimensional Gaussian distribution with zero mean and the variance-covariance matrix $\Sigma$.

PROOF. Without loss of generality, we only give the proof for the case when $d=m=1$. The essential part of the present proof is in the fact that we can use the integration by parts operation repeatedly. First, the formula for $\mathbf{E}\left[\Phi_{0}\right]$ is the direct result of calculation. Second, the expectation of the first term of $\Phi_{1}$ is given by

$$
\begin{aligned}
& \mathbf{E}\left[\left\{\left.\frac{\partial \phi^{(\varepsilon)}}{\partial \varepsilon}\right|_{\varepsilon=0}\left(g_{1}\right)+\partial \phi\left(g_{1}\right) g_{2}\right\} I_{\mathcal{B}}\left(g_{1}\right)\right] \\
& \quad=\int_{\mathcal{B}}\left\{\left.\frac{\partial \phi^{(\varepsilon)}}{\partial \varepsilon}\right|_{\varepsilon=0}(x)+\partial \phi^{(\varepsilon)}(x) \mathbf{E}\left[g_{2} \mid g_{1}=x\right]\right\} n\left[x \mid 0, \Sigma_{g_{1}}\right] d x .
\end{aligned}
$$

As for the expectation of $\phi^{(0)}\left(g_{1}\right) \partial I_{\mathcal{B}}\left(g_{1}\right) g_{2}$, we notice that $\phi^{(0)}\left(g_{1}\right) g_{2} \in \boldsymbol{D}^{\infty}(\boldsymbol{R})$. Then by using the integration by parts formula for Wiener functionals, we have

$$
\begin{aligned}
\mathbf{E}\left[\phi^{(0)}\left(g_{1}\right) \partial I_{\mathcal{B}}\left(g_{1}\right) g_{2}\right] & =\mathbf{E}\left[G(w) I_{\mathcal{B}}\left(g_{1}\right)\right] \\
& =\mathbf{E}\left[\mathbf{E}\left[G(w) \mid g_{1}=x\right] I_{\mathcal{B}}\left(g_{1}\right)\right] \\
& =\int_{\mathcal{B}} \mathbf{E}\left[G(w) \mid g_{1}=x\right] n\left[x \mid 0, \Sigma_{g_{1}}\right] d x \\
& \equiv \int_{\mathcal{B}} p_{1}(x) d x
\end{aligned}
$$

for a smooth Wiener functional $G(w)$. In order to obtain an explicit representation of $p_{1}(x)$, we set $\mathcal{B}_{x}=(-\infty, x]$. Then we have

$$
\begin{aligned}
\mathbf{E}\left[\phi^{(0)}\left(g_{1}\right) \partial I_{\mathcal{B}_{x}}\left(g_{1}\right) g_{2}\right] & =\int_{-\infty}^{\infty} \phi^{(0)}(y) E\left[g_{2} \mid g_{1}=y\right] \partial I_{\mathcal{B}_{x}}(y) n\left[y \mid 0, \Sigma_{g_{1}}\right] d y \\
& =-\int_{-\infty}^{\infty} \phi^{(0)}(y) E\left[g_{2} \mid g_{1}=y\right] \delta_{x}(y) n\left[y \mid 0, \Sigma_{g_{1}}\right] d y \\
& =-\phi^{(0)}(x) E\left[g_{2} \mid g_{1}=x\right] n\left[x \mid 0, \Sigma_{g_{1}}\right],
\end{aligned}
$$

where $\delta_{x}(y)$ denotes the delta function of $y$ at $x$. By differentiating the above term 
with respect to $x$, we have

$$
p_{1}(x)=\frac{\partial}{\partial x}\left[-\phi^{(0)}(x) \mathbf{E}\left[g_{2} \mid g_{1}=x\right] n\left[x \mid 0, \Sigma_{g_{1}}\right]\right] .
$$

By adding two terms, we have the explicit formula for $\mathbf{E}\left[\Phi_{1}\right]$. Third, we shall derive an explicit representation for $\mathbf{E}\left[\Phi_{2}\right]$, which is more complicated. For this purpose, we write it as

$$
\mathbf{E}\left[\Phi_{2}\right]=\int_{\mathcal{B}} p_{21}(x) d x+\int_{\mathcal{B}} p_{22}(x) d x+\int_{\mathcal{B}} p_{23}(x) d x,
$$

where $p_{2 i}(i=1,2,3)$ corresponds to each line of $\Phi_{2}$. The first term $p_{21}(x)$ can be calculated directly as $\mathbf{E}\left[\Phi_{1}\right]$ by using the integration by parts formula and is given by

$$
\begin{aligned}
p_{21}(x)=\frac{\partial}{\partial x}[- & \left.\frac{\partial \phi^{(\varepsilon)}}{\partial \varepsilon}\right|_{\varepsilon=0}(x) \mathbf{E}\left[g_{2} \mid g_{1}=x\right] \\
& \left.\left.+\partial \phi^{(\varepsilon)}(x) \mathbf{E}\left[g_{2}^{2} \mid g_{1}=x\right]\right\} n\left[x \mid 0, \Sigma_{g_{1}}\right]\right] .
\end{aligned}
$$

For the second term, we only need the standard differentiation, and it is given by

$$
\begin{aligned}
p_{22}(x)= & {\left[\left.\frac{1}{2} \frac{\partial^{2} \phi^{(\varepsilon)}}{\partial \varepsilon^{2}}\right|_{\varepsilon=0}(x)+\left\{\left.\frac{\partial^{2} \phi^{(\varepsilon)}(x)}{\partial x \partial \varepsilon}\right|_{\varepsilon=0}\right\} \mathbf{E}\left[g_{2} \mid g_{1}=x\right]\right.} \\
& \left.+\partial \phi^{(0)}(x) \mathbf{E}\left[g_{3} \mid g_{1}=x\right]+\frac{1}{2} \partial^{2} \phi^{(0)}(x) \mathbf{E}\left[g_{2}^{2} \mid g_{1}=x\right]\right] n\left[x \mid 0, \Sigma_{g_{1}}\right] .
\end{aligned}
$$

In order to derive $p_{23}(x)$, first we need an expression of the second-order generalized derivatives of Wiener functional $\mathbf{E}\left[\frac{1}{2} \phi^{(0)}\left(g_{1}\right) \partial^{2} I_{\mathcal{B}}\left(g_{1}\right) g_{2}^{2}\right]$. By taking $\mathcal{B}=\mathcal{B}_{x}=(-\infty, x]$ and using the integration by parts formulas for Wiener functionals, we have

$$
\begin{aligned}
\mathbf{E}\left[\frac{1}{2}\right. & \left.\phi^{(0)}\left(g_{1}\right) \partial^{2} I_{\mathcal{B}_{x}}\left(g_{1}\right) g_{2}^{2}\right] \\
& =\int_{-\infty}^{\infty} \partial^{2} I_{\mathcal{B}_{x}}(y)\left\{\frac{1}{2} \phi^{(0)}(y) \mathbf{E}\left[g_{2}^{2} \mid g_{1}=y\right] n\left[y \mid 0, \Sigma_{g_{1}}\right]\right\} d y \\
& =\frac{\partial}{\partial x} \int_{-\infty}^{\infty} \delta_{x}(y)\left\{\frac{1}{2} \phi^{(0)}(y) \mathbf{E}\left[g_{2}^{2} \mid g_{1}=y\right] n\left[y \mid 0, \Sigma_{g_{1}}\right]\right\} d y \\
& =\frac{\partial}{\partial x}\left\{\frac{1}{2} \phi^{(0)}(x) \mathbf{E}\left[g_{2}^{2} \mid g_{1}=x\right] n\left[x \mid 0, \Sigma_{g_{1}}\right]\right\} \\
& =\int_{-\infty}^{x} \frac{\partial^{2}}{\partial y^{2}}\left\{\frac{1}{2} \phi^{(0)}(y) \mathbf{E}\left[g_{2}^{2} \mid g_{1}=y\right] n\left[y \mid 0, \Sigma_{g_{1}}\right]\right\} d y .
\end{aligned}
$$


For the term of $\mathbf{E}\left[\phi^{(0)}\left(g_{1}\right) \partial I_{\mathcal{B}}\left(g_{1}\right) g_{3}\right]$, we obtain

$$
\mathbf{E}\left[\phi^{(0)}\left(g_{1}\right) \partial I_{\mathcal{B}_{x}} g_{3}\right]=\int_{-\infty}^{x} \frac{\partial}{\partial y}\left\{-\phi^{(0)}(y) \mathbf{E}\left[g_{3} \mid g_{1}=y\right] n\left[y \mid 0, \Sigma_{g_{1}}\right]\right\} d y .
$$

Hence, $p_{23}(x)$ is given by

$$
\begin{aligned}
p_{23}(x)= & \frac{\partial^{2}}{\partial x^{2}}\left\{\frac{1}{2} \phi^{(0)}(x) \mathbf{E}\left[g_{2}^{2} \mid g_{1}=x\right] n\left[x \mid 0, \Sigma_{g_{1}}\right]\right\} \\
& +\frac{\partial}{\partial x}\left\{-\phi^{(0)}(x) \mathbf{E}\left[g_{3} \mid g_{1}=x\right] n\left[x \mid 0, \Sigma_{g_{1}}\right]\right\} .
\end{aligned}
$$

Finally, by collecting and rearranging each term of $p_{21}(x), p_{22}(x)$, and $p_{23}(x)$, we have the result.

If we take a particular function $\phi^{(\varepsilon)}(x)$, we can derive the corresponding formulas in the asymptotic expansion. Here we give simple examples when $d=$ $m=1$ for the illustration. When we take $\phi^{(\varepsilon)}(x) \equiv 1$ and $\mathcal{B}=(-\infty, x]$, then we have an asymptotic expansion of the distribution function, which is given by

$$
\begin{aligned}
P\left(\left\{X_{T}^{(\varepsilon)} \leq x\right\}\right) \sim & \int_{-\infty}^{x} n\left[y \mid 0, \Sigma_{g_{1}}\right] d y \\
& +\varepsilon \int_{-\infty}^{x}\left[-\frac{\partial}{\partial y} \mathbf{E}\left[g_{2} \mid g_{1}=y\right] n\left[y \mid 0, \Sigma_{g_{1}}\right]\right] d y \\
& +\varepsilon^{2} \int_{-\infty}^{x}\left[\frac{1}{2} \frac{\partial^{2}}{\partial y^{2}}\left\{\mathbf{E}\left[g_{2}^{2} \mid g_{1}=y\right] n\left[y \mid 0, \Sigma_{g_{1}}\right]\right\}\right. \\
& \left.\quad+\frac{\partial}{\partial y}\left\{-\mathbf{E}\left[g_{3} \mid g_{1}=y\right] n\left[y \mid 0, \Sigma_{g_{1}}\right]\right\}\right] d y+\cdots .
\end{aligned}
$$

Also, for the the pay-off function of European call options, we set $\phi^{(\varepsilon)}(x)=x+y$ for a constant $y$ and $\mathcal{B}=[-y, \infty)$. Then we have

$$
\begin{aligned}
\mathbf{E}\left[(x+y)^{+}\right] \sim & \int_{-y}^{\infty}(y+x) n\left[x \mid 0, \Sigma_{g_{1}}\right] d x \\
& +\varepsilon \int_{-y}^{\infty} x\left[-\frac{\partial}{\partial x} \mathbf{E}\left[g_{2} \mid g_{1}=x\right] n\left[x \mid 0, \Sigma_{g_{1}}\right]\right] d x \\
& +\varepsilon^{2} \int_{-y}^{\infty} x\left[\frac{\partial}{\partial x}\left\{-\mathbf{E}\left[g_{3} \mid g_{1}=x\right] n\left[x \mid 0, \Sigma_{g_{1}}\right]\right\}\right. \\
& \left.\quad+\frac{1}{2} \frac{\partial^{2}}{\partial x^{2}}\left\{\mathbf{E}\left[g_{2}^{2} \mid g_{1}=x\right] n\left[x \mid 0, \Sigma_{g_{1}}\right]\right\}\right] d x+\cdots
\end{aligned}
$$

These results we have obtained are equivalent to the formulas for the European call options previously reported by Takahashi (1999). In fact, the formulae in 
Theorem 3.4 are equivalent to those obtained by the characteristic functions and their the Fourier inversions originally obtained by Fujikoshi, Morimune, Kunitomo and Taniguchi (1982). They have been extensively used in Kunitomo and Takahashi $(1995,2001)$ and Takahashi (1999).

As a more complicated application, we consider the problem arising in the valuation of the Asian options mainly because it illustrates a wide applicability of our approach in mathematical finance. The explicit formulas have been derived in Section 3.2 of Takahashi (1999). The terminal pay-off for the Asian options is dependent on

$$
A_{T}^{(\varepsilon)}=\int_{0}^{T} f\left(S_{s}^{(\varepsilon)}\right) d s
$$

where $f(\cdot)$ is a smooth function, which is in $\boldsymbol{C}^{\infty}\left(\boldsymbol{R}^{d} \rightarrow \boldsymbol{R}\right)$ and $S_{s}^{(\varepsilon)}$ satisfies (3.1)-(3.3). In this case we take $A_{T}^{(0)}=\int_{0}^{T} f\left(S_{S}^{(0)}\right) d s$ and we need to derive the asymptotic expansion of the random variable $F_{T}^{(\varepsilon)}=(1 / \varepsilon)\left[A_{T}^{(\varepsilon)}-A_{T}^{(0)}\right]$. By using a formal Taylor expansion, an asymptotic expansion of the random variable $F_{T}^{(\varepsilon)}$ can be written as

$$
F_{T}^{(\varepsilon)} \sim g_{1 T}(A)+\varepsilon g_{2 T}(A)+\varepsilon^{2} g_{3 T}(A)+\cdots,
$$

where $g_{n T}(A)(n \geq 1)$ are defined by $g_{n T}(n \geq 1)$ in Theorem 3.1 as

$$
g_{n T}(A)=\int_{0}^{T} \sum_{\substack{k=1 \\ m_{1}+\cdots+m_{k}=n}}^{n}\left[\frac{1}{k !} \sum_{l_{1}, \ldots, l_{k}=1}^{d} \partial_{l_{1}, \ldots, l_{k}}^{k} f\left(S_{s}^{(0)}, s\right) \prod_{j=1}^{k} g_{m_{j}, s}^{\left(l_{j}\right)}\right] d s
$$

and $m_{j}(j=1, \ldots, k)$ are positive integers.

By using the smoothness condition of $f(\cdot), S_{T}^{(\varepsilon)} \in \boldsymbol{D}^{\infty}\left(\boldsymbol{R}^{d}\right)$, and $g_{1 s}, g_{2 s}$, $g_{3 s}, \ldots \in \boldsymbol{D}^{\infty}\left(\boldsymbol{R}^{d}\right)$, we see that $F_{T}^{(\varepsilon)}$ has an asymptotic expansion, which is in $\boldsymbol{D}^{\infty}(\boldsymbol{R})$ as $\varepsilon \downarrow 0$ with $g_{k T}(A) \in \boldsymbol{D}^{\infty}(\boldsymbol{R})(k=1,2, \ldots)$. The Malliavin covariance of $F_{T}^{(\varepsilon)}$, which is denoted as $\sigma_{\mathrm{MC}}\left(F_{T}^{(\varepsilon)}\right)$, is given by

$$
\sigma_{\mathrm{MC}}\left(F_{T}^{(\varepsilon)}\right)=\int_{0}^{T}\left|\left\{\int_{u}^{T} \partial f\left(S_{s}^{(\varepsilon)}\right) Y_{s}^{(\varepsilon)} d s\right\} Y_{u}^{(\varepsilon)-1} \sigma\left(S_{u}^{(\varepsilon)}, u\right)\right|^{2} d u .
$$

If we define $\eta_{c}^{\varepsilon}(A)$ as before by

$$
\begin{aligned}
\eta_{c}^{\varepsilon}(A)=c \int_{0}^{T} \mid & \int_{u}^{T} \partial f\left(S_{s}^{(\varepsilon)}\right) Y_{s}^{(\varepsilon)} d s Y_{u}^{(\varepsilon)-1} \sigma\left(S_{u}^{(\varepsilon)}, u\right) \\
& -\left.\int_{u}^{T} \partial f\left(S_{s}^{(0)}\right) Y_{s} d s Y_{u}^{-1} \sigma\left(S_{u}^{(0)}, u\right)\right|^{2} d u,
\end{aligned}
$$

then we have the corresponding results as for $\eta_{c}^{\varepsilon}(A)$ instead of $\eta_{c}^{\varepsilon}$ exactly in the same way. As before, we need to make use of Lemma A.2 in the Appendix. 
Consequently, we can apply Theorem 2.1 to $\psi\left(\eta_{c}^{\varepsilon}(A)\right) \phi\left(F_{T}^{(\varepsilon)}\right) I_{\mathcal{B}}\left(F_{T}^{(\varepsilon)}\right)$, and the same results as in Theorem 3.3 and Corollary 3.1 can be obtainable for $F_{T}^{(\varepsilon)}$ if we use the next assumption instead of Assumption I.

AsSUMPTION I'. For any $T>0$,

$$
\Sigma_{g_{1}}(A)=\int_{0}^{T}\left|\left\{\int_{u}^{T} \partial f\left(S_{s}^{(0)}\right) Y_{s} d s\right\} Y_{u}^{-1} \sigma\left(S_{u}^{(0)}, u\right)\right|^{2} d u>0 .
$$

THEOREM 3.5. Under the assumptions in (3.1)-(3.3), the smoothness of $f(\cdot)$ in $C^{\infty}$ and Assumption $\mathrm{I}^{\prime}, \psi\left(\eta_{c}^{\varepsilon}(A)\right) \phi^{(\varepsilon)}\left(F_{T}^{(\varepsilon)}\right) I_{\mathcal{B}}\left(F_{T}^{(\varepsilon)}\right)$ has an asymptotic expansion

$$
\psi\left(\eta_{c}^{\varepsilon}(A)\right) \phi^{(\varepsilon)}\left(F_{T}^{(\varepsilon)}\right) I_{\mathcal{B}}\left(F_{T}^{(\varepsilon)}\right) \sim \Phi_{0}+\varepsilon \Phi_{1}+\cdots
$$

in $\tilde{D}^{-\infty}(\boldsymbol{R})$ as $\varepsilon \downarrow 0$, where $\Phi_{0}, \Phi_{1}, \ldots$ are determined by the formal Taylor expansion with respect to $F_{T}^{(\varepsilon)}\left(\sim g_{1 T}(A)+\varepsilon g_{2 T}(A)+\cdots\right)$, and $\phi^{(\varepsilon)}(\cdot), \psi(\cdot)$ and $I_{B}(\cdot)$ are defined as in Theorem 3.3.

COROLLARY 3.2. Under the assumptions in (3.1)-(3.3), the smoothness of $f(\cdot)$ in $\boldsymbol{C}^{\infty}$ and Assumption $\mathrm{I}^{\prime}$, an asymptotic expansion of $\mathbf{E}\left[\phi^{(\varepsilon)}\left(F_{T}^{(\varepsilon)}\right) I_{\mathcal{B}}\left(F_{T}^{(\varepsilon)}\right)\right]$ is given by

$$
\begin{aligned}
\mathbf{E}\left[\phi^{(\varepsilon)}\left(F_{T}^{(\varepsilon)}\right) I_{\mathscr{B}}\left(F_{T}^{(\varepsilon)}\right)\right] & \sim \mathbf{E}\left[\psi\left(\eta_{c}^{\varepsilon}(Z)\right) \phi^{(\varepsilon)}\left(F^{(\varepsilon)}\right) I_{\mathcal{B}}\left(F^{(\varepsilon)}\right)\right] \\
& \sim \mathbf{E}\left[\Phi_{0}\right]+\varepsilon \mathbf{E}\left[\Phi_{1}\right]+\cdots
\end{aligned}
$$

as $\varepsilon \downarrow 0$, where $\phi^{(\varepsilon)}(\cdot), \psi(\cdot), \Phi_{j}(j \geq 0)$ and $\mathcal{B}$ are defined as in Theorem 3.3.

REMARK. The general valuation problem of financial contingent claims including the European options and the Asian options in the Black-Scholes economy can be simply defined as finding its "fair" value at financial markets. Let $V(T)$ be the pay-off of a contingent claim at terminal period $T$. Then the standard martingale theory in financial economics predicts that the fair price of $V(T)$ at time $t(0 \leq t<T)$ should be given by

$$
V_{t}(T)=\mathbf{E}_{t}\left[\exp \left[-\int_{t}^{T} r\left(S_{v}^{(\varepsilon)}, v\right) d v\right] V(T)\right],
$$

where $\mathbf{E}_{t}[\cdot]$ stands for the conditional expectation operator given the information available at $t$ with respect to the equivalent martingale measure $Q$. Then we can expand the expected value with respect to the parameter $\varepsilon$ and use the formulas in Theorem 3.4. Takahashi $(1995,1999)$ has already given many asymptotic expansion formulas for the examples we have mentioned in this section including the Asian options and others when $r$ is a positive constant. In this case, the conditions in (3.2) on the drift terms are automatically satisfied. 
4. Validity in the term structure model of interest rates. Let $(\Omega, \mathcal{F}, Q$, $\left.\left\{\mathcal{F}_{t}\right\}_{t \in[0, T]}\right)$ be the filtered probability space with $T<+\infty$ and $\left\{w_{t}^{i} ; i=1, \ldots, m\right\}$ are independent Brownian motions with respect to $\left\{\mathcal{F}_{t}\right\}$. Let also $\Gamma_{T}=\{(s, t) \mid 0 \leq$ $s \leq t \leq T\}$ be a compact set in $\boldsymbol{R}^{2}$. We shall consider a class of random fields $\bar{f}^{(\varepsilon)}(s, t): \Gamma_{T} \rightarrow \boldsymbol{R}$ which are adapted with respect to $\left\{\widetilde{F}_{t}\right\}$ and satisfy the stochastic integral equation

$$
\begin{aligned}
f^{(\varepsilon)}(s, t)= & f(0, t)+\varepsilon^{2} \int_{0}^{s}\left[\sum_{i=1}^{m} \sigma^{i}\left(f^{(\varepsilon)}(v, t), v, t\right)\right. \\
& \left.\times \int_{v}^{t} \sigma^{i}\left(f^{(\varepsilon)}(v, y), v, y\right) d y\right] d v \\
& +\varepsilon \int_{0}^{s} \sum_{i=1}^{m} \sigma^{i}\left(f^{(\varepsilon)}(v, t), v, t\right) d w_{v}^{i} .
\end{aligned}
$$

We note that there are integrals with respect to the maturity parameter in the drift term involving $\left\{\sigma^{i}\left(f^{(\varepsilon)}(v, y), v, y\right)(i=1, \ldots, m),(v, y) \in \Gamma_{T}\right\}$. We can construct such integrals recursively by considering the discretized versions with respect to the maturity argument as

$$
\int_{v}^{t} \sigma^{i}\left(f^{(\varepsilon)}\left(v, \psi_{n^{\prime}}(y)\right), v, \psi_{n^{\prime}}(y)\right) d y
$$

for $0 \leq v \leq s \leq y \leq t \leq T$, where $\psi_{n^{\prime}}(s)=(k+1) T / 2^{n^{\prime}}$ if $s \in\left(k T / 2^{n^{\prime}}\right.$, $\left.(k+1) T / 2^{n^{\prime}}\right]\left(k=0, \ldots, 2^{n^{\prime}}-1 ; n^{\prime} \geq 1\right)$. Then we can make the solution $f^{(\varepsilon)}\left(s, \psi_{n^{\prime}}(t)\right)$ of (4.1) to be adapted with respect to $\mathcal{F}_{s}(0 \leq s \leq t \leq T)$. Actually, by using the standard argument in stochastic analysis, we can further discretize the volatility functions as $\sigma^{i}\left(f^{(\varepsilon)}\left(\phi_{n}(v), \psi_{n^{\prime}}(y)\right), \phi_{n}(v), \psi_{n^{\prime}}(y)\right)$ for $0 \leq v \leq y \leq T$, where $\phi_{n}(v)=k T / 2^{n}$ if $v \in\left[k T / 2^{n},(k+1) T / 2^{n}\right)\left(k=0, \ldots, 2^{n}-1 ; n \geq\right.$ $\left.n^{\prime} \geq 1\right)$. Then by using a real polynomial function $p_{1}\left(x_{1}, x_{2}, \ldots, x_{2^{n}}\right)$, the first part of the solution of the discretized version can be written as

$$
f^{n, n^{\prime}(\varepsilon)}\left(s, \tau\left(n^{\prime}\right)\right)=p_{1}\left(\left[h_{1}\right](w),\left[h_{2}\right](w), \ldots,\left[h_{\phi_{n}^{*}(s)+1}\right](w), \cdot\right)
$$

for $0 \leq s \leq \tau\left(n^{\prime}\right)$, where $\tau\left(n^{\prime}\right)=T / 2^{n^{\prime}}$ and $\phi_{n}^{*}(s)=\phi_{n}(s) / \tau(n)$. Also by using real polynomial functions $p_{k}(\cdot)\left(k=2, \ldots, 2^{n^{\prime}}\right)$, we have a recursive representation as

$$
f^{n, n^{\prime}(\varepsilon)}\left(s, k \tau\left(n^{\prime}\right)\right)=p_{k}\left(\left[h_{1}\right](w), \ldots,\left[h_{\phi_{n}^{*}(s)+1}\right](w), \cdot, p_{k-1}(\cdot), \ldots, p_{1}(\cdot)\right)
$$

for $k=2, \ldots, 2^{n^{\prime}}$ and $0 \leq s \leq k \tau\left(n^{\prime}\right)$. Hence the solution of the discretized version of (4.1) can be represented as polynomial functions of $\left[h_{1}\right](w),\left[h_{2}\right](w), \ldots$, $\left[h_{2^{n}}\right](w)$.

Returning to (4.1), we make the following assumptions on the volatility functions in this section. 
Assumption II. The volatility functions $\sigma^{i}\left(f^{(\varepsilon)}(s, t), s, t\right)(i=1, \ldots, m)$ are nonnegative, measurable, bounded, and smooth in the first argument, and all derivatives are bounded uniformly in $\varepsilon$. The initial forward rates $f(0, t)$ are also Lipschitz continuous with respect to $t$.

Assumption III. For any $0<s \leq t \leq T$,

$$
\Sigma(s, t)=\int_{0}^{s} \sum_{i=1}^{m}\left[\sigma^{(0) i}(v, t)\right]^{2} d v>0,
$$

where $\sigma^{(0) i}(v, t)=\left.\sigma^{i}\left(f^{(\varepsilon)}(v, t), v, t\right)\right|_{\varepsilon=0}$.

The conditions stated in Assumption II exclude the possibility of explosions for the solution of (4.1). Assumption III ensures the key condition of nondegeneracy of the Malliavin covariance in our problem, which is essential for the validity of the asymptotic expansion approach for the forward rate processes. Under Assumption II we can get the stochastic expansions of the forward rates and spot interest rates processes. The starting point of our discussion is a simplified version of the result by Morton (1989) on the existence of the solution of the stochastic integral equation (4.1) for forward rate processes.

THEOREM 4.1 [Morton (1989)]. Under Assumption II, there exists a jointly continuous process $\left\{f^{(\varepsilon)}(s, t), 0 \leq s \leq t \leq T\right\}$ satisfying (4.1) with $\varepsilon=1$. There exists only one solution of (4.1) with $\varepsilon=1$.

In the rest of this section we often refer to the case of $m=1$ whenever we can avoid complicated notation and the proofs are as if it is the general case without loss of generality. For that purpose we use the convention $w_{v}=w_{v}^{1}$ and $\sigma\left(f^{(\varepsilon)}(s, t), s, t\right)=\sigma^{1}\left(f^{(\varepsilon)}(s, t), s, t\right)$ when $m=1$. We construct the completion of the polynomial functions of $p_{k}\left(\left[h_{1}\right](w),\left[h_{2}\right](w), \ldots,\left[h_{2^{n}}\right](w)\right)(k=$ $\left.1, \ldots, 2^{n^{\prime}}\right)$. With a fixed $n^{\prime}$ we will abuse the notation slightly and denote the resulting totality of polynomials and the totality of smooth functionals as $\boldsymbol{P}(\boldsymbol{R})$ and $\boldsymbol{S}(\boldsymbol{R})$, respectively, in this section. If we denote the resulting forward processes as $f^{n^{\prime}(\varepsilon)}\left(s, \psi_{n^{\prime}}(t)\right)$, then for any $p>1$ we have

$$
\boldsymbol{E}\left[\sup _{0 \leq s \leq t \leq T}\left|f^{(\varepsilon)}(s, t)-f^{n^{\prime}(\varepsilon)}\left(s, \psi_{n^{\prime}}(t)\right)\right|^{p}\right] \rightarrow 0
$$

as $n^{\prime} \rightarrow+\infty$ by using the standard arguments in stochastic analysis. [See Chapters IV and V of Ikeda and Watanabe (1989).] Hence in the rest of this section we consider the sequence of $\left\{f^{n^{\prime}(\varepsilon)}\left(s, \psi_{n^{\prime}}(t)\right)\right\}$ as if they were $\left\{f^{(\varepsilon)}(s, t)\right\}$ to avoid the resulting tedious arguments. The $k$ th order $H$-derivative $(k \geq 1)$ of the forward rate process $f^{n^{\prime}(\varepsilon)}\left(s, \psi_{n^{\prime}}(t)\right) \in \boldsymbol{S}(\boldsymbol{R})$ is denoted as $D^{k} f^{(\varepsilon)}(s, t) \in \boldsymbol{S}\left(\boldsymbol{H}^{\otimes k} \otimes \boldsymbol{R}\right)$.

We summarize the first major result in this section on the sequence of forward processes $\left\{f^{(\varepsilon)}(s, t)\right\}$ as the next theorem. The proof is the result of lengthy arguments on the higher order $H$-derivatives of $\left\{f^{(\varepsilon)}(s, t)\right\}$ given in the Appendix. 
THEOREM 4.2. Suppose Assumption II hold for the forward rate processes following (4.1). Then for any $\varepsilon \in(0,1]$ and $(s, t) \in \Gamma_{T}, f^{(\varepsilon)}(s, t) \in \boldsymbol{D}^{\infty}(\boldsymbol{R})$.

Next we consider the asymptotic behavior of a functional,

$$
F^{(\varepsilon)}(s, t)=\frac{1}{\varepsilon}\left[f^{(\varepsilon)}(s, t)-f^{(0)}(0, t)\right]
$$

as $\varepsilon \rightarrow 0$. Then the $H$-derivative of $F^{(\varepsilon)}(s, t)$ can be represented as

$$
D_{h} F^{(\varepsilon)}(s, t)=\int_{0}^{s} Y^{(\varepsilon)}(s, t) Y^{(\varepsilon)-1}(v, t) C^{(\varepsilon)}(v, t) d v
$$

where the stochastic process $\left\{Y^{(\varepsilon)}(s, t), 0 \leq s \leq t \leq T\right\}$ is defined as the solution of the stochastic integral equations

$$
\begin{aligned}
Y^{(\varepsilon)}(s, t)=1+\varepsilon^{2} \int_{0}^{s}\left[\partial \sigma\left(f^{(\varepsilon)}(v, t), v, t\right)\right. & \\
& \left.\times \int_{v}^{t} \sigma\left(f^{(\varepsilon)}(v, y), v, y\right) d y\right] Y^{(\varepsilon)}(v, t) d v \\
& +\varepsilon \int_{0}^{s} \partial \sigma\left(f^{(\varepsilon)}(v, t), v, t\right) Y^{(\varepsilon)}(v, t) d w_{v}
\end{aligned}
$$

and

$$
\begin{aligned}
C^{(\varepsilon)}(v, t)= & \sigma\left(f^{(\varepsilon)}(v, t), v, t\right) \dot{h}_{v} \\
& +\varepsilon \sigma\left(f^{(\varepsilon)}(v, t), v, t\right) \int_{v}^{t} \partial \sigma\left(f^{(\varepsilon)}(v, y), v, y\right) D_{h} f^{(\varepsilon)}(v, y) d y .
\end{aligned}
$$

We notice that the coefficients of $Y^{(\varepsilon)}(s, t)$ on the right-hand side of (4.6) are bounded under Assumption II. Hence for any $1<p<+\infty, 0<\varepsilon \leq 1$, we have $\mathbf{E}\left[\left|Y^{(\varepsilon)}(s, t)\right|^{p}\right]+\mathbf{E}\left[\left|Y^{(\varepsilon)-1}(s, t)\right|^{p}\right]<+\infty$. The proof of this result has been given in Kunitomo and Takahashi (2001). By rearranging terms in the integrands of (4.5), we have the representation

$$
D_{h} F^{(\varepsilon)}(s, t)=\int_{0}^{s} v_{s, t}^{(\varepsilon, 1)}(u) \dot{h}_{u} d u,
$$

where

$$
\begin{aligned}
v_{s, t}^{(\varepsilon, 1)}(u)= & Y^{(\varepsilon)}(s, t) Y^{(\varepsilon)-1}(u, t) \sigma\left(f^{(\varepsilon)}(u, t), u, t\right) \\
& +\varepsilon \int_{u}^{s} Y^{(\varepsilon)}(s, t) Y^{(\varepsilon)-1}(v, t) \sigma\left(f^{(\varepsilon)}(v, t), v, t\right) \\
& \times\left(\int_{v}^{t} \partial \sigma\left(f^{(\varepsilon)}(v, y), v, y\right) \xi_{v, y}^{(\varepsilon, 1)}(u) d y\right) d v
\end{aligned}
$$


and $\left\{\xi_{v, y}^{(\varepsilon, 1)}(u)\right\}$ are defined by $\left\{\xi_{v, y}^{(1,1)}(u)\right\}$ of (A.8) in the Appendix by replacing $(1,1)$ with $(\varepsilon, 1)$. Hence the Malliavin covariance of $F^{(\varepsilon)}(s, t), \sigma_{\mathrm{MC}}\left(F^{(\varepsilon)}(s, t)\right)$, is obtained by

$$
\left\langle D F^{(\varepsilon)}, D F^{(\varepsilon)}\right\rangle_{H}=\int_{0}^{s}\left|v_{s, t}^{(\varepsilon, 1)}(u)\right|^{2} d u
$$

Let

$$
\begin{gathered}
\eta_{c}^{(\varepsilon)}(s, t)=c \int_{0}^{s} \mid \varepsilon\left(\int_{u}^{s} Y^{(\varepsilon)}(s, t) Y^{(\varepsilon)-1}(v, t) \sigma\left(f^{(\varepsilon)}(v, t), v, t\right)\right. \\
\left.\quad \times \int_{v}^{t} \partial \sigma\left(f^{(\varepsilon)}(v, y), v, y\right) \xi_{v, y}^{(\varepsilon, 1)}(u) d y d v\right)\left.\right|^{2} d u \\
+c \int_{0}^{s} \mid Y^{(\varepsilon)}(s, t) Y^{(\varepsilon)-1}(u, t) \sigma\left(f^{(\varepsilon)}(u, t), u, t\right) \\
-\left.\sigma\left(f^{(0)}(u, t), u, t\right)\right|^{2} d u,
\end{gathered}
$$

for a positive constant $c>0$. We notice that the condition in Assumption III is equivalent to the nondegeneracy condition of (4.8) because $Y^{(0)}(v, t)=1$ for $(v, t) \in \Gamma_{T}$. Again by using the similar arguments as Lemma A.2 in the Appendix, for $(s, t) \in \Gamma_{T}$ and any $k \geq 1$,

$$
\lim _{\varepsilon \rightarrow 0} \varepsilon^{-k} P\left\{\left|\eta_{c}^{(\varepsilon)}(s, t)\right|>\frac{1}{2}\right\}=0 .
$$

Then by a similar argument as Theorem 3.2 in Section 3, we shall obtain a truncated version of the nondegeneracy condition of the Malliavin covariance for the spot interest rates and forward rates processes, which is the key step to show the validity of the asymptotic expansion approach.

THEOREM 4.3. Under Assumptions II and III, the Malliavin covariance $\sigma\left(F^{(\varepsilon)}(s, t)\right)$ of $F^{(\varepsilon)}(s, t)$ is uniformly nondegenerate in the sense that there exists $c_{0}>0$ such that for any $c>c_{0}$ and any $p>1$,

$$
\sup _{0<\varepsilon \leq 1} \mathbf{E}\left[I\left(\left|\eta_{c}^{(\varepsilon)}\right| \leq 1\right)\left(\sigma_{\mathrm{MC}}\left(F^{(\varepsilon)}(s, t)\right)\right)^{-p}\right]<+\infty
$$

where $I(\cdot)$ is the indicator function.

Hence the validity of the asymptotic expansions of the distribution function or the density function of instantaneous spot rate, and forward rates can be obtained under Assumption II and Assumption III because we have proved that a set of conditions in Theorem 2.1 are satisfied.

We now return to the general case where $m \geq 1$. By expanding the Wiener functional $F^{(\varepsilon)}(s, t)$, we can write

$$
F^{(\varepsilon)}(s, t) \sim A_{1}(s, t)+\varepsilon A_{2}(s, t)+\cdots .
$$


The coefficients in the asymptotic expansion can be obtained by applying a formal Taylor expansion and $A_{n}(s, t)(n \geq 1)$ are given by

$$
A_{1}(s, t)=\sum_{i=1}^{m} \int_{0}^{s} \sigma^{i}\left(f^{(0)}(0, t), v, t\right) d w_{v}^{i},
$$

and for $n \geq 2$,

$$
\begin{aligned}
& A_{n}(s, t)=\sum_{i^{\prime}=1}^{m} \int_{0}^{s}\left[\sum_{\substack{j_{1}+\cdots+j_{k}=k+l \\
j_{1}^{\prime}+\cdots+j^{\prime}, l, l^{\prime} \geq 0 \\
k+k^{\prime}+l+l^{\prime}+2=n}}^{j_{1}^{\prime}+l^{\prime}} \frac{1}{k ! k^{\prime} !} \partial^{k} \sigma^{i^{\prime}}\left(f^{(0)}(v, t), v, t\right) \prod_{i^{*}=1}^{k} A_{j_{i}}(v, t)\right. \\
& \left.\times \int_{v}^{t} \partial^{k^{\prime}} \sigma^{i^{\prime}}\left(f^{(0)}(v, y), v, y\right) \prod_{i=1}^{k} A_{j_{i}}(v, y) d y\right] d v \\
& +\sum_{i^{\prime}=1}^{m} \int_{0}^{s}\left[\sum_{\substack{k, l \geq 0 \\
k+l+1=n}}^{j_{1}+\cdots+j_{k}=k+l} \frac{1}{k !} \partial^{k} \sigma^{i^{\prime}}\left(f^{(0)}(v, t), v, t\right) \prod_{i^{*}=1}^{k} A_{j_{i}^{*}}(v, t)\right] d w_{v}^{i^{\prime}} .
\end{aligned}
$$

Some of these formulas have been previously obtained by Kunitomo and Takahashi (1995, 2001). By applying similar arguments, which are actually quite tedious, we can show that the $L_{p}$-boundedness of any order $H$-derivatives of $A_{n}(s, t)$ for any $0 \leq s \leq t \leq T$ and integers $n \geq 1$. Then we conclude that $A_{n}(s, t) \in \boldsymbol{D}^{\infty}(\boldsymbol{R})$ for any $n \geq 1$ and summarize the result as the next theorem.

THEOREM 4.4. Under Assumption II, $F^{(\varepsilon)}(s, t)$ is in $\boldsymbol{D}^{\infty}(\boldsymbol{R})$ and has an asymptotic expansion,

$$
F^{(\varepsilon)}(s, t) \sim A_{1}(s, t)+\varepsilon A_{2}(s, t)+\cdots
$$

as $\varepsilon \downarrow 0$ and $A_{1}(s, t), A_{2}(s, t), \ldots \in \boldsymbol{D}^{\infty}(\boldsymbol{R})$.

We notice that the Malliavin covariance is nondegenerate because $\Sigma(s, t)$ is nondegenerate, which is in turn the variance of $A_{1}(s, t)$. Then we have the Gaussian random variable as the leading term in (4.13) and we can use the same method as in Section 3 to derive the asymptotic expansion of the expected values of random variables. By applying the corresponding one as Theorem 2.1 for $\boldsymbol{D}^{\infty}(\boldsymbol{R})$, it has a proper asymptotic expansion as $\varepsilon \rightarrow 0$ in $\tilde{\boldsymbol{D}}^{-\infty}(\boldsymbol{R})$. Hence we obtain the next result.

THEOREM 4.5. Under Assumptions II and III, an asymptotic expansion of $\mathbf{E}\left[\phi^{(\varepsilon)}\left(F^{(\varepsilon)}\right) I_{\mathcal{B}}\left(F^{(\varepsilon)}\right)\right]$ is given by

$$
\begin{aligned}
\mathbf{E}\left[\phi^{(\varepsilon)}\left(F^{(\varepsilon)}\right) I_{\mathcal{B}}\left(F^{(\varepsilon)}\right)\right] & \sim \mathbf{E}\left[\psi\left(\eta_{c}^{\varepsilon}\right) \phi^{(\varepsilon)}\left(F^{(\varepsilon)}\right) I_{\mathcal{B}}\left(F^{(\varepsilon)}\right)\right] \\
& \sim \mathbf{E}\left[\Phi_{0}\right]+\varepsilon \mathbf{E}\left[\Phi_{1}\right]+\cdots
\end{aligned}
$$


as $\varepsilon \downarrow 0$, where $\Phi_{j}(j \geq 0)$ are obtained by a formal Taylor expansion of the lefthand side in the expectation operator with respect to $F^{(\varepsilon)}(s, t)$ and $\psi\left(\eta_{c}^{\varepsilon}\right), \phi^{(\varepsilon)}(\cdot)$, and $I_{B}(\cdot)$ are defined as in Theorem 3.3.

Also it is straightforward to obtain the similar nondegeneracy conditions of the Malliavin covariance for the discounted coupon bond price process and the average interest rate process. We note that the discount bond price process is given by (1.5). Then using (4.1) and Itô's lemma, we can consider the stochastic process $G^{(\varepsilon)}(t, T, p)=\left[P^{(\varepsilon)}(t, T)\right]^{p}$ for any integer $p>1$, which is the solution of the stochastic integral equation

$$
\begin{aligned}
& G^{(\varepsilon)}(t, T, p) \\
& =G^{(\varepsilon)}(0, T, p) \\
& \quad+\int_{0}^{t}\left[p r^{(\varepsilon)}(v)\right. \\
& \left.\quad+\frac{p^{2}-p}{2} \varepsilon^{2} \sum_{i=1}^{m}\left(\int_{t}^{T} \sigma^{i}\left(f^{(\varepsilon)}(v, y), v, y\right) d y\right)^{2}\right] G^{(\varepsilon)}(v, T, p) d v \\
& \quad+\sum_{i=1}^{m} \int_{0}^{t}(-p \varepsilon)\left[\int_{t}^{T} \sigma^{i}\left(f^{(\varepsilon)}(v, y), v, y\right) d y\right] G^{(\varepsilon)}(v, T, p) d w_{v}^{i} .
\end{aligned}
$$

Hence by using the fact that $E\left[\left|r^{(\varepsilon)}(t)\right|^{p}\right]<+\infty$ for any $p>1$ and $0 \leq s \leq t \leq T$ under Assumption II, we have $\mathbf{E}\left[\left|P^{(\varepsilon)}(s, t)\right|^{p}\right]<+\infty$. Then we can investigate the properties of the $H$-derivatives on the set of discount bond price processes as for the forward rate and spot rate processes we have discussed. Because the essential arguments are the same, we only report the result.

THEOREM 4.6. Under Assumption II for the forward rate processes, for any $\varepsilon \in(0,1]$ and $0 \leq t \leq T, P^{(\varepsilon)}(t, T)$ is in $\boldsymbol{D}^{\infty}(\boldsymbol{R})$ and has an asymptotic expansion

$$
P^{(\varepsilon)}(t, T) \sim P(t, T)+\varepsilon B_{1}(t, T)+\varepsilon^{2} B_{2}(t, T)+\cdots
$$

as $\varepsilon \downarrow 0$ and $B_{1}(t, T), B_{2}(t, T), \ldots \in D^{\infty}(\boldsymbol{R})$, where $P(t, T)\left(=P^{(0)}(t, T)\right)$, $B_{j}(t, T)(j \geq 1)$ are obtained by a formal Taylor expansion of $P^{(\varepsilon)}(t, T)$ through (1.5), (4.4) and (4.13).

More generally, the valuation problem of many interest rates based contingent claims in the complete market can be simply defined as to find the "fair" value of a function of a series of bond prices at financial markets. Then the fair price of $V(T)$ at time $t(0 \leq t<T)$ should be given by

$$
V_{t}(T)=\mathbf{E}_{t}\left[\exp \left[-\int_{t}^{T} r^{(\varepsilon)}(s) d s\right] V(T)\right],
$$


where $V(T)$ is the pay-off of a contingent claim at the terminal period $T$ and $\mathbf{E}_{t}[\cdot]$ stands for the conditional expectation operator given the information available at $t$ with respect to the equivalent martingale measure $Q$. Because we can derive an asymptotic expansion of the spot interest rate $r^{(\varepsilon)}(s)$, it is straightforward to obtain the fair value of interest rates-based contingent claims.

For instance, most interest rates-based contingent claims can be regarded as functionals of bond prices with different maturities. Let $\left\{c_{j}, j=1, \ldots, k\right\}$ be a sequence of nonnegative coupon payments and $\left\{T_{j}, j=1, \ldots, k\right\}$ be a sequence of payment periods satisfying the condition $0 \leq t<T_{1} \leq \cdots \leq T_{k} \leq T \leq+\infty$. Then the price of the coupon bond with coupon payments $\left\{c_{j}, j=1, \ldots, k\right\}$ at $t$ is given by

$$
P_{k,\left\{T_{j}\right\},\left\{c_{j}\right\}}^{(\varepsilon)}(t)=\sum_{j=1}^{k} c_{j} P^{(\varepsilon)}\left(t, T_{j}\right),
$$

where $\left\{P^{(\varepsilon)}\left(t, T_{j}\right), j=1, \ldots, k\right\}$ are the prices of zero coupon bonds with different maturities. The normalized random variable for the call options on the discounted coupon bond at the initial period $t=0$ is given by

$$
\begin{aligned}
& R_{k,\left\{T_{j}\right\},\left\{c_{j}\right\}}^{(\varepsilon)}(t) \\
&=\frac{1}{\varepsilon}\left\{\exp \left[-\int_{0}^{t} r^{(\varepsilon)}(s) d s\right]\left[P_{k,\left\{T_{j}\right\},\left\{c_{j}\right\}}^{(\varepsilon)}(t)-K\right]\right. \\
&\left.-\left[\sum_{j=1}^{k} c_{j} P\left(0, T_{j}\right)-K P(0, t)\right]\right\},
\end{aligned}
$$

where $0<t<T_{1} \leq \cdots \leq T_{k}$ and $K$ is a fixed real constant. This random variable has an intuitive interpretation in financial applications. Its meaning and the related additional assumptions for practical applications have been discussed in Section 3 of Kunitomo and Takahashi (2001). By using (1.5) and (4.16), the first order $H$-derivative of $R_{k,\left\{T_{j}\right\},\left\{c_{j}\right\}}^{(\varepsilon)}(t)$ can be represented as

$$
\begin{aligned}
D_{h}\left[R_{k,\left\{T_{j}\right\},\left\{c_{j}\right\}}^{(\varepsilon)}(t)\right] \\
=-\exp \left[-\int_{0}^{t} r^{(\varepsilon)}(s) d s\right]\left[P_{k,\left\{T_{j}\right\},\left\{c_{j}\right\}}^{(\varepsilon)}(t)-K\right] \int_{0}^{t} D_{h}\left[F^{(\varepsilon)}(s, s)\right] d s \\
\quad-\exp \left[-\int_{0}^{t} r^{(\varepsilon)}(s) d s\right]\left[\sum_{j=1}^{k} c_{j} P^{(\varepsilon)}\left(t, T_{j}\right) \int_{T}^{T_{j}} D_{h}\left[F^{(\varepsilon)}(t, u)\right] d u\right],
\end{aligned}
$$

where $F^{(\varepsilon)}(t, u)$ is defined by (4.4).

From this expression we can obtain a simplified representation of the first-order $H$-derivative in this case as before. Hence we can obtain the asymptotic expansion 
of the expected pay-off value of a coupon bond if we use the condition ensuring the nondegeneracy of the Malliavin covariance. The proof of the next theorem is similar to those in the previous results.

ASSUMPTION IV. For any $0<t<T_{1} \leq \cdots \leq T_{k}$

$$
\Sigma_{g_{1}}(k)=\int_{0}^{t} \sigma_{g_{1}}^{*}(v) \boldsymbol{\sigma}_{g_{1}}^{*^{\prime}}(v) d v>0
$$

where $\sigma_{g_{1}}^{*}(v)=-\left[\sum_{j=1}^{k} c_{j} P\left(0, T_{j}\right)-K P(0, t)\right] \sigma_{t}^{(0)}(v)-\sum_{i=1}^{k} c_{j} P\left(0, T_{j}\right) \times$ $\sigma_{t, T_{j}}^{(0)}(v)$, and $\sigma_{t}^{(0)}(v)$ and $\sigma_{t, T_{j}}^{(0)}(v)$ are $1 \times m$ vectors such that $\sigma_{t}^{(0)}(v)=$ $\left[\int_{v}^{t} \sigma_{i}^{(0)}(v, y) d y\right]$ and $\sigma_{t, T_{j}}^{(0)}(v)=\left[\int_{t}^{T_{j}} \sigma_{i}^{(0)}(v, u) d u\right]$.

THEOREM 4.7. Under Assumptions II and IV, an asymptotic expansion of $\mathbf{E}\left[\phi^{(\varepsilon)}\left(R_{k,\left\{T_{j}\right\},\left\{c_{j}\right\}}^{(\varepsilon)}(t)\right) I_{\mathscr{B}}\left(R_{k,\left\{T_{j}\right\},\left\{c_{j}\right\}}^{(\varepsilon)}(t)\right)\right]$ is given by

$$
\begin{aligned}
\mathbf{E}\left[\phi^{(\varepsilon)}\right. & \left.\left(R_{k,\left\{T_{j}\right\},\left\{c_{j}\right\}}^{(\varepsilon)}(t)\right) I_{\mathcal{B}}\left(R_{k,\left\{T_{j}\right\},\left\{c_{j}\right\}}^{(\varepsilon)}(t)\right)\right] \\
& \sim \mathbf{E}\left[\psi\left(\eta_{c}^{\varepsilon}\right) \phi^{(\varepsilon)}\left(R_{k,\left\{T_{j}\right\},\left\{c_{j}\right\}}^{(\varepsilon)}(t)\right) I_{\mathcal{B}}\left(R_{k,\left\{T_{j}\right\},\left\{c_{j}\right\}}^{(\varepsilon)}(t)\right)\right] \\
& \sim \mathbf{E}\left[\Phi_{0}^{*}\right]+\varepsilon \mathbf{E}\left[\Phi_{1}^{*}\right]+\cdots
\end{aligned}
$$

as $\varepsilon \downarrow 0$, where $\Phi_{j}^{*}(j \geq 0)$ are obtained by a formal Taylor expansion of the lefthand side in the expectation operator, and $\psi\left(\eta_{c}^{\varepsilon}\right), \phi^{(\varepsilon)}(\cdot)$ and $I_{\mathcal{B}}(\cdot)$ are defined as in Theorem 3.3.

We briefly mention two examples of interest rates-based contingent claims. The pay-off function of the call bond options with coupon payments $\left\{c_{j}, j=\right.$ $1, \ldots, k\}$ at $\left\{T_{j}, j=1, \ldots, k\right\}$ can be written as $V^{(1)}(T)=\left[P_{k,\left\{T_{j}\right\},\left\{c_{j}\right\}}^{(\varepsilon)}(T)-K\right]^{+}$, where $K$ is a fixed strike price. In this case we can take $\phi^{(\varepsilon)}(x)=x+y$ for a constant $y$ and $\mathcal{B}=[-y, \infty)$. As another example we should mention the pay-off function of the call options on average interest rates, which is given by $V^{(2)}(T)=\left[\frac{1}{T} \int_{0}^{T} L^{\tau}(t) d t-K\right]^{+}$, where the yield of a zero coupon bond at $t$ with time to maturity of $\tau\left(0<t<t+\tau<T_{k}\right)$ years is given by $L^{\tau}(t)=$ $\left[1 / P^{(\varepsilon)}(t, t+\tau)-1\right] / \tau$. Then we can apply the asymptotic expansion method with some additional assumptions. For these two examples and others, Kunitomo and Takahashi $(1995,2001)$ have derived more explicit formulas of the asymptotic expansions in details.

REMARK. We should mention that we can use the equivalence between the formulas by the expected values of the generalized Wiener functionals and those derived by using the simple inversion technique for the characteristic functions of random variables as we have discussed in Section 3. 
5. Numerical examples. In this section we will present numerical examples in order to illustrate the usefulness of approximations obtained by the asymptotic expansion method we have discussed. There have been many examples and some of them have been already reported by Kunitomo and Takahashi (1995, 2001) and Takahashi (1999). As an example of the Black-Scholes economy, we give some numerical results on the average call options for the square root process and the log-normal process for the underlying asset prices. Under the equivalent martingale measure, we assume that the processes of the onedimensional underlying asset are given by

$$
\begin{aligned}
& d S^{1\left(\varepsilon_{1}\right)}=(r-q) S^{1\left(\varepsilon_{1}\right)} d t+\varepsilon_{1} \sigma\left(S^{1\left(\varepsilon_{1}\right)}\right)^{1 / 2} d w_{t}, \\
& d S^{2\left(\varepsilon_{2}\right)}=(r-q) S^{2\left(\varepsilon_{2}\right)} d t+\varepsilon_{2} S^{2\left(\varepsilon_{2}\right)} d w_{t},
\end{aligned}
$$

where $\varepsilon_{1}, \varepsilon_{2}, \sigma$ are parameters, and $r$ and $q$ denote the risk-free interest rate and a dividend yield (we assume both are positive constants), respectively, and $w_{t}$ denotes the one-dimensional Brownian motion. The theoretical value of the average (or Asian) call options at time 0 should be given by

$$
\mathbf{E}_{0}\left[\exp (-r T) \max \left\{\frac{1}{T} \int_{0}^{T} S_{u}^{i\left(\varepsilon_{i}\right)} d u-K, 0\right\}\right], \quad i=1,2,
$$

where $K$ is the strike price. The terminal pay-off in this example is a special case of (3.19) and then we can apply Corollary 3.2 to this case.

Note. In this example the volatility function is not smooth at the origin and we need to use a smoothed version of the square root process at the origin for the mathmatical point of view. It is possible to show that the smoothing and the truncation by a large threshold value do not make significant differences and the effects are negligible in the small disturbance asymptotic theory.

In Tables 1-5 we have calculated the differences between the Monte Carlo value and the second-order approximations based on asymptotic expansions. The values in Tables 4 and 5 are calculated in terms of the basis points except the percentage points (\%). Difference (bp) and difference rate (\%) are calculated by the deviations from the Monte Carlo results in (1). The values in the last columns were calculated by setting $\varepsilon_{1}=\varepsilon_{2}=0.0$ ( or $\varepsilon=0.0$ ), which could be regarded as the zeroth order approximations.

Table 1 shows the numerical values of the average call options for the square root processes of the underlying asset which represents an equity index with no dividend. We take the spot price $S_{0}=40.00$, the risk-free rate $r=5 \%$, the parameter $\sigma=10.00$ and the six month maturity date. The parameters $\varepsilon_{1}$ and $\varepsilon_{2}$ were set so that the instantaneous volatility is equivalent to the corresponding volatility of the $30 \% \log$-normal process (i.e., $\varepsilon_{1}=0.189737$ and $\varepsilon_{2}=0.3$ ). 
TABLE 1

Average call options on equity square root processes

\begin{tabular}{llll}
\hline Strike price & \multicolumn{1}{c}{$\mathbf{4 5}$} & \multicolumn{1}{c}{$\mathbf{4 0}$} & \multicolumn{1}{c}{$\mathbf{3 5}$} \\
\hline (1) Monte Carlo & 0.5221 & 2.1758 & 5.6468 \\
(2) Stochastic expansion & 0.5228 & 2.1788 & 5.6516 \\
Difference & 0.00078 & 0.00301 & 0.00482 \\
Difference rate, \% & 0.15 & 0.14 & 0.09 \\
Value when $\varepsilon_{1}=0.0$ & 0.0 & 0.4917 & 5.3683 \\
\hline
\end{tabular}

Tables 2 and 3 are the numerical values of the average call options on the foreign exchange rate example when the underlying assets follow square root processes and the log-normal process, respectively. In this example we take the spot price $S_{0}=100.00$ and regard $r$ as the risk-free interest rate in Japan and $q$ as the riskfree interest rate in the U.S., and we set 3\% and 5\%, respectively. In Tables 2 and 3 the spot price, the six month maturity date and the parameters $\varepsilon_{1}$ and $\varepsilon_{2}$ were set so that the instantaneous variance at time 0 are equivalent to $10 \%$ volatility of the $\log$-normal process (i.e., $\varepsilon_{1}=0.158114$ and $\varepsilon_{2}=0.1$ ).

For the purpose of comparison, the values by the Monte Carlo simulations are also given, which are based on 500,000 trials implemented in each case. We also report the value based on the PDE numerical method developed by $\mathrm{He}$ and Takahashi (2000) for Table 3. The approximations given by the asymptotic expansion are those from the approximations up to the second order for Tables 1-3 where they are based on the total approximations consisting of the basic deterministic terms, the first-order terms based on the Gaussian distribution and the additional second-order terms based on the non-Gaussian adjustments. In Tables 1-3 it is apparent that we have enough accuracy of approximations for financial applications by the asymptotic expansion approach. More details of this example and other examples in the Black-Scholes economy have been discussed by Takahashi (1999).

TABLE 2

Average call options on FX square root processes

\begin{tabular}{llll}
\hline Strike price & \multicolumn{1}{c}{$\mathbf{1 0 5}$} & \multicolumn{1}{c}{$\mathbf{1 0 0}$} & \multicolumn{1}{c}{$\mathbf{9 5}$} \\
\hline (1) Monte Carlo & 0.1721 & 1.3625 & 4.6858 \\
(2) Stochastic expansion & 0.1730 & 1.3654 & 4.6931 \\
Difference & 0.00090 & 0.00286 & 0.00730 \\
Difference rate, \% & 0.52 & 0.21 & 0.16 \\
Value when $\varepsilon_{1}=0.0$ & 0.0 & 0.0 & 4.4346 \\
\hline
\end{tabular}


TABLE 3

Average options on FX log-normal process

\begin{tabular}{lccc}
\hline Strike price & $\mathbf{1 0 5}$ & $\mathbf{1 0 0}$ & $\mathbf{9 5}$ \\
\hline (1) Monte Carlo simulation method & 0.1840 & 1.3682 & 4.6793 \\
(2) Stochastic expansion & 0.1830 & 1.3660 & 4.6800 \\
Difference rate, \% & -0.54 & -0.16 & 0.01 \\
(3) Finite difference (Crank-Nicholson method) & 0.1831 & 1.3656 & 4.6788 \\
Difference rate, $\%$ & -0.49 & -0.19 & -0.01 \\
Value when $\varepsilon_{2}=0.0$ & 0.0 & 0.0 & 4.4346 \\
\hline
\end{tabular}

NOTE. Since the final formulas in our approximations are analytical which are simple functions of the Gaussian distribution functions and some low-order Hermitian polynomials, the computation running times are negligible by any computational standards. Also at the suggestion of a referee we have added the deterministic values in the last column by setting $\varepsilon_{1}=\varepsilon_{2}=0.0(\varepsilon=0.0$ for the interest rate based contingent claims), which could be regarded as the zeroth order approximations.

As an example of the non-Markovian term structure model of interest rates, we give an example of swaptions in the HJM term structure model. For simplicity of exposition, we assume that the instantaneous forward rate processes $\left\{f^{(\varepsilon)}(s, t)\right\}$ have one-factor volatility function as $\sigma\left(f^{(\varepsilon)}(s, t), s, t\right)=\left[f^{(\varepsilon)}(s, t)\right]^{\beta}$, where $0 \leq \beta \leq 1$ and $m=1$ in (4.1).

NOTE. We have used the truncated version of this forward rate process when $0<\beta \leq 1$ because the original process theoretically could have explosive solutions. For the Gaussian forward rate case other numerical valuation methods have been known, but we report the results for comparative purposes. See Kunitomo and Takahashi (2001) for the details.

Tables 4 and 5 show the numerical values of the call options of a swap contract (the swaption) for the case when $\beta=0$ and $\varepsilon=0.01$ (100 bp) and the case when

TABLE 4

Swaption (Gaussian case)

\begin{tabular}{lccccc}
\hline Fixed rate \% & $\mathbf{7 . 1 8}$ & $\mathbf{6 . 1 6}$ & $\mathbf{5 . 1 3}$ & $\mathbf{4 . 1 0}$ & $\mathbf{3 . 0 8}$ \\
\hline (1) Monte Carlo & 774.6 & 518.2 & 315.0 & 170.9 & 81.3 \\
(2) Stochastic expansion & 774.8 & 518.5 & 315.1 & 171.15 & 81.2 \\
Difference (bp) & 0.28 & 0.36 & 0.16 & 0.36 & 0.03 \\
Difference rate, \% & 0.04 & 0.07 & 0.05 & 0.21 & 0.04 \\
Value when $\varepsilon=0.0$ & 689.1 & 344.5 & 0.0 & 0.0 & 0.0 \\
\hline
\end{tabular}


TABLE 5

Swaption (log-normal case)

\begin{tabular}{lrrrrr}
\hline Fixed rate \% & $\mathbf{7 . 1 8}$ & $\mathbf{6 . 1 6}$ & $\mathbf{5 . 1 3}$ & $\mathbf{4 . 1 0}$ & $\mathbf{3 . 0 8}$ \\
\hline (1) Monte Carlo & 814.1 & 542.6 & 312.3 & 140.2 & 39.6 \\
(2) Stochastic expansion & 819.1 & 546.5 & 315.1 & 143.3 & 42.3 \\
Difference (bp) & 5.0 & 3.9 & 2.8 & 3.1 & 2.7 \\
Difference rate, \% & 0.6 & 0.7 & 0.9 & 2.2 & 6.8 \\
Value when $\varepsilon=0.0$ & 689.1 & 344.5 & 0.0 & 0.0 & 0.0 \\
\hline
\end{tabular}

$\beta=1$ and $\varepsilon=0.2(20 \%)$, respectively. In both cases we consider that the term of the underlying interest swap is five years, the time to expiration is also five years, and we set $\tau=1$ (year), $T=5, T_{1}=6, \ldots, T_{5}=10$ and $k=5$. The present term structure at $t=0$ is assumed to be flat at $5 \%$ per year and we took $c_{j}=S \tau(j=$ $1, \ldots, 4), c_{5}=1+S \tau, S=\left[P(0, T)-P\left(0, T_{5}\right)\right] / \tau \sum_{j=1}^{5} P\left(0, T_{j}\right)=0.05171$ and $K=1.00$. In this example the theoretical value of swaption at time 0 should be given by

$$
\mathbf{E}_{0}\left[\exp \left[-\int_{0}^{T} r^{(\varepsilon)}(s) d s\right] \max \left\{\sum_{j=1}^{k} c_{j} P^{(\varepsilon)}\left(T, T_{j}\right)-K, 0\right\}\right] .
$$

Then we can apply Theorem 4.7 to this case. We have used the approximations based on the asymptotic expansions and examine their accuracy by Monte Carlo results for all cases. We have given the numerical results for the out-of-the-money case $(S=5.171 \% \times 0.8,5.171 \% \times 0.6)$, at-the-money case $(S=5.171 \%)$, and in-the-money case $(S=5.171 \% \times 1.2,5.171 \% \times 1.4)$. From Tables 4 and 5 we find that the differences in the option values by the asymptotic expansion approach for the Gaussian forward rates case are very small, and the differences of the option values between the approximations and the Monte Carlo results for the geometric Brownian forward rates case become slightly larger due to the nonGaussianity of the underlying forward rates and the spot rates. Nonetheless, we still have enough acuracy in our approximations for financial applications since the differences between the approximations and the corresponding Monte Carlo results are usually within 3 bp in most cases. Kunitomo and Takahashi (2001) have discussed more examples in the HJM term structure of the interest rates model.

6. Concluding remarks. This paper gives the mathematical validity of the asymptotic expansion approach for the valuation problem of financial contingent claims when the underlying forward rates follow a general class of continuous Itô processes in the HJM term structure of the interest rates model and the underlying asset prices follow a general class of diffusion processes in the Black-Scholes economy. Our method, called the small disturbance asymptotic theory, can be applicable to a wide range of valuation problems of financial contingent claims. 
Some of them have been discussed by Kunitomo and Takahashi $(1995,2001)$ and Takahashi (1999).

Since the asymptotic expansion approach can be justified rigorously by the Watanabe-Yoshida theory on the Malliavin calculus in stochastic analysis, it is not an ad hoc method to give numerical approximations. In Section 5 of this paper and in our previous papers [Kunitomo and Takahashi $(1995,2001)$ and Takahashi (1999)], we have illustrated that the approximations we have obtained via the asymptotic expansion method can be satisfactory in many cases for practical purposes as well.

\section{APPENDIX}

In this Appendix we give some mathematical details omitted in Sections 3 and 4. First we present two inequalities which are useful to show that the truncation by the random variable $\eta_{c}^{\varepsilon}$ of (3.13) in Section 3 is negligible in probability under the assumptions of (3.1)-(3.4) when we derive the asymptotic expansion of random variables.

Note. The present proof of Lemma A.1, which is simpler than our original one, is due to the referee. Actually we only need the conditions given by (3.2)-(3.3) with $k=1$ for Lemma A.1.

LEMMA A.1. There exist positive constants $a_{i}(i=1,2)$ independent of $\varepsilon$ such that

$$
P\left(\sup _{0 \leq s \leq T}\left[\left|S_{s}^{(\varepsilon)}-S_{s}^{(0)}\right|+\left|Y_{s}^{(\varepsilon)}-Y_{s}\right|\right]>a_{0}\right) \leq a_{1} \exp \left(-a_{2} \varepsilon^{-2}\right)
$$

for all $a_{0}>0$, where $S_{t}^{(\varepsilon)}$ and $Y_{t}^{(\varepsilon)}$ are defined by (3.1) and (3.11), respectively.

ProOF. Let $Z_{t}^{(\varepsilon)}=\left(S_{t}^{(\varepsilon) 1}, \ldots, S_{t}^{(\varepsilon) d}, Y_{t}^{(\varepsilon) 11}, \ldots, Y_{t}^{(\varepsilon) d d}\right)^{\prime}$ be a $d_{1} \times 1$ state vector with $d_{1}=d(d+1)$. By using (3.1) for $S_{t}^{(\varepsilon)}$ and (3.11) for $Y_{t}^{(\varepsilon)}, Z_{t}^{(\varepsilon)}[=$ $\left(Z_{t}^{(\varepsilon) i}\right)$ ] follows the stochastic differential equation in the form of

$$
Z_{T}^{(\varepsilon) i}=Z_{0}^{i}+\int_{0}^{T} b^{i}\left(Z_{s}^{(\varepsilon) i}, s\right) d s+\varepsilon \sum_{j=1}^{m} \int_{0}^{T} \omega^{i j}\left(Z_{s}^{(\varepsilon)}, s\right) d w_{s}^{j},
$$

where $b\left(Z_{s}^{(\varepsilon)}, s\right)=\left(b^{i}\left(Z_{s}^{(\varepsilon)}, s\right)\right)$ and $\omega\left(Z_{s}^{(\varepsilon)}, s\right)=\left(\omega^{i j}\left(Z_{s}^{(\varepsilon)}, s\right)\right)$ are $R^{d_{1}} \times$ $[0, T] \rightarrow R^{d_{1}}$ and $R^{d_{1}} \times[0, T] \rightarrow R^{d_{1}} \otimes R^{m}$ Borel measurable functions which are smooth with respect to $Z_{s}^{(\varepsilon)}$. By using the Lipschitz continuity, there exists a positive constant $K_{3}$ such that

$$
\left|Z_{t}^{(\varepsilon)}-Z_{t}^{(0)}\right| \leq K_{3} \int_{0}^{t}\left|Z_{s}^{(\varepsilon)}-Z_{s}^{(0)}\right| d s+\sup _{0 \leq s \leq t}\left|\int_{0}^{s} \varepsilon \omega\left(Z_{u}^{(\varepsilon)}, u\right) d w_{u}\right|
$$


Furthermore, by using the Gronwall inequality,

$$
\sup _{0 \leq s \leq T}\left|Z_{s}^{(\varepsilon)}-Z_{s}^{(0)}\right| \leq \sup _{0 \leq s \leq T}\left|\int_{0}^{s} \omega\left(Z_{u}^{(\varepsilon)}, u\right) d w_{u}\right| \varepsilon e^{K_{3} T} .
$$

If we can assume that for the $d_{1} \times 1$ vector $\theta$ there exists $A(>0)$ such that

$$
\sup _{|\theta|=1,0 \leq t \leq T}\left\langle\theta, \omega\left(Z_{t}^{(\varepsilon)}, t\right) \omega^{*}\left(Z_{t}^{(\varepsilon)}, t\right) \theta\right\rangle \leq A<\infty,
$$

we can apply the standard large deviation result given by Theorem 4.2.1 in Stroock and Varadhan (1979). Hence for any $a_{0}>0$ we have

$$
\begin{aligned}
& P\left(\left\{\sup _{0 \leq t \leq T}\left|Z_{t}^{(\varepsilon)}-Z_{t}^{(0)}\right|>a_{0}\right\}\right) \\
& \quad \leq P\left(\left\{\sup _{0 \leq t \leq T}\left|\int_{0}^{T} \omega\left(Z_{t}^{(\varepsilon)}, t\right) d w_{t}\right|>\frac{a_{0}}{\varepsilon e^{K_{3} T}}\right\}\right) \\
& \quad \leq 2 d_{1} \exp \left\{-\frac{a_{0}^{2} e^{-2 K_{3} T}}{2 A d_{1} T} \varepsilon^{-2}\right\} .
\end{aligned}
$$

When $A$ is not bounded, let a stopping time be $\tau=\inf _{0 \leq t \leq T}\left\{\left|Z_{t}^{(\varepsilon)}-Z_{t}^{(0)}\right|>a_{0}\right\}$ for any $a_{0}>0$. Then

$$
\begin{aligned}
& P\left(\left\{\sup _{0 \leq t \leq T}\left|Z_{t}^{(\varepsilon)}-Z_{t}^{(0)}\right|>a_{0}\right\}\right) \\
& \quad \leq P\left(\left\{\tau<T, \sup _{0 \leq t \leq \tau}\left|\int_{0}^{T} \omega\left(Z_{t}^{(\varepsilon)}, t\right) d w_{t}\right|>\frac{a_{0}}{\varepsilon e^{K_{3} T}}\right\}\right) \\
& \quad \leq 2 d_{1} \exp \left(-\frac{a_{0}^{2} e^{-2 K_{3} T}}{2 A d_{1} T} \varepsilon^{-2}\right),
\end{aligned}
$$

where

$$
\sup _{|\theta|=1,\left|Z_{t}^{(\varepsilon)}-Z_{t}^{(0)}\right| \leq a_{0}}\left\langle\theta, \omega\left(Z_{t}^{(\varepsilon)}, t\right) \omega^{*}\left(Z_{t}^{(\varepsilon)}, t\right) \theta\right\rangle \leq A<\infty .
$$

LEMMA A.2. Let the random variable $\eta_{c}^{\varepsilon}$ be defined by (3.13). Then for any $c>0 \eta_{c}^{\varepsilon}$ is $O(1)$ in $\boldsymbol{D}^{\infty}(\boldsymbol{R})$ and for $c_{0}>0$ there exist some positive constants $c_{i}(i=2,3,4)$, such that

$$
P\left(\left\{\left|\eta_{c}^{\varepsilon}\right|>c_{0}\right\}\right) \leq c_{2} \exp \left(-c_{3} \varepsilon^{-c_{4}}\right) .
$$

ProOF. We notice that

$$
\begin{aligned}
\left|\eta_{c}^{\varepsilon}\right| & =c \int_{0}^{T}\left|Y_{T}^{(\varepsilon)} Y_{s}^{(\varepsilon)-1} \sigma\left(S_{s}^{(\varepsilon)}, s\right)-Y_{T} Y_{s}^{-1} \sigma\left(S_{s}^{(0)}, s\right)\right|^{2} d s \\
& \leq c T \sup _{0 \leq s \leq T}\left|Y_{T}^{(\varepsilon)} Y_{s}^{(\varepsilon)-1} \sigma\left(S_{s}^{(\varepsilon)}, s\right)-Y_{T} Y_{s}^{-1} \sigma\left(S_{s}^{(0)}, s\right)\right|^{2} .
\end{aligned}
$$


The set $\left\{\left|\eta_{c}^{\varepsilon}\right|>c_{0}\right\}$ is included in $\left\{\sup _{0 \leq s \leq T} \mid Y_{T}^{(\varepsilon)} Y_{s}^{(\varepsilon)-1} \sigma\left(S_{s}^{(\varepsilon)}, s\right)-Y_{T} Y_{s}^{-1} \times\right.$ $\left.\sigma\left(S_{s}^{(0)}, s\right) \mid>\left(c_{0} / c T\right)^{1 / 2}\right\}$. By setting a constant $c_{1}=\sqrt{c_{0} /(9 c T)}$, we have

$$
\begin{aligned}
\left\{\left|\eta_{c}^{\varepsilon}\right|>c_{0}\right\} \subset & \left\{\sup _{0 \leq s \leq T}\left|Y_{T} Y_{s}^{-1}\right|\left|\sigma\left(S^{(\varepsilon)}, s\right)-\sigma\left(S_{s}^{(0)}, s\right)\right|>c_{1}\right\} \\
& \cup\left\{\sup _{0 \leq s \leq T}\left|Y_{s}^{(\varepsilon)-1}\right|\left|\sigma\left(S_{s}^{(\varepsilon)}, s\right)\right|\left|Y_{T}^{(\varepsilon)}-Y_{T}\right|>c_{1}\right\} \\
& \cup\left\{\sup _{0 \leq s \leq T}\left|Y_{T}\right|\left|\sigma\left(S_{s}^{(\varepsilon)}, s\right)\right|\left|Y_{s}^{(\varepsilon)-1}-Y_{s}^{-1}\right|>c_{1}\right\} .
\end{aligned}
$$

Then by using Lemma A.1 because of the boundedness of $\left|Y_{T} Y_{s}^{-1}\right|$ and the smoothness of $\sigma\left(S_{S}^{(\varepsilon)}, s\right)$, we have the result.

Next we shall give the proof of Theorem 4.2 in Section 4 by using three lemmas and some additional derivations.

PROOF OF THEOREM 4.2. Without loss of generality we only give the proof when $m=1$. First we consider first-order derivatives $\left\{D f^{(1)}(s, t)\right\}$. For any $h \in H$, we successively define a sequence of random variables $\left\{\xi^{(l)}(s, t) ;(s, t) \in \Gamma_{T}\right.$, $l \geq 1\}$ by the integral equation

$$
\begin{aligned}
\xi^{(l+1)}(s, t)= & \int_{0}^{s}\left[\partial \sigma\left(f^{(1)}(v, t), v, t\right) \int_{v}^{t} \sigma\left(f^{(1)}(v, y), v, y\right) d y \xi^{(l)}(v, t)\right] d v \\
& +\int_{0}^{s}\left[\sigma\left(f^{(1)}(v, t), v, t\right) \int_{v}^{t} \partial \sigma\left(f^{(1)}(v, y), v, y\right) \xi^{(l)}(v, y) d y\right] d v \\
& +\int_{0}^{s} \partial \sigma\left(f^{(1)}(v, t), v, t\right) \xi^{(l)}(v, t) d w_{v} \\
& +\int_{0}^{s} \sigma\left(f^{(1)}(v, t), v, t\right) \dot{h}_{v} d v
\end{aligned}
$$

where the initial condition is given by $\xi^{(0)}(s, t)=0$. Then we have the next result by using the standard method of stochastic analysis. The proof is given in Kunitomo and Takahashi (1995).

LEMMA A.3. For any $p>1$ and $0 \leq s \leq t \leq T, \mathbf{E}\left[\left|\xi^{(l)}(s, t)\right|^{p}\right]<\infty(l>1)$, and there exists a positive constant $M_{4}$ such that

$$
\mathbf{E}\left[\sup _{0 \leq u \leq s}\left|\xi^{(l+1)}(u, t)-\xi^{(l)}(u, t)\right|^{2}\right] \leq \frac{1}{(l+1) !}\left[M_{4}(t+1) s\right]^{l+1} .
$$

As $l \rightarrow \infty$,

$$
\sup _{0 \leq s \leq t \leq T} \mathbf{E}\left[\sup _{0 \leq u \leq s}\left|\xi^{(l+1)}(u, t)-\xi^{(l)}(u, t)\right|^{2}\right] \rightarrow 0
$$


Using Lemma A.3 and the Chebyshev inequality, we have

$$
\sum_{l=1}^{\infty} P\left\{\sup _{0 \leq u \leq s \leq t}\left|\xi^{(l+1)}(u, s)-\xi^{(l)}(u, s)\right|>\frac{1}{2^{l}}\right\} \leq \sum_{l=1}^{\infty} \frac{1}{l !}\left[4 M_{4}(T+1) T\right]^{l}<+\infty .
$$

Then by the Borel-Cantelli lemma, the sequence of random variables $\left\{\xi^{(l)}(s, t)\right\}$ converges uniformly on $(s, t) \in \Gamma_{T}$. Hence we have established the existence of the $H$-derivative of $f^{(1)}(s, t)$, which is given by the solution of the stochastic integral equation

$$
\begin{aligned}
& D_{h} f^{(1)}(s, t)=\int_{0}^{s}\left[\partial \sigma\left(f^{(1)}(v, t), v, t\right)\right. \\
&\left.\times \int_{v}^{t} \sigma\left(f^{(1)}(v, y), v, y\right) d y D_{h} f^{(1)}(v, t)\right] d v \\
&+\int_{0}^{s}\left[\sigma\left(f^{(1)}(v, t), v, t\right)\right. \\
&\left.\times \int_{v}^{t} \partial \sigma\left(f^{(1)}(v, y), v, y\right) D_{h} f^{(1)}(v, y) d y\right] d v \\
&+\int_{0}^{s} \partial \sigma\left(f^{(1)}(v, t), v, t\right) D_{h} f^{(1)}(v, t) d w_{v} \\
&+\int_{0}^{s} \sigma\left(f^{(1)}(v, t), v, t\right) \dot{h}_{v} d v
\end{aligned}
$$

We note that for the spot rate process $\left\{r^{(\varepsilon)}(t)\right\}$ the $H$-derivative can be well defined by

$$
D_{h} r^{(\varepsilon)}(t)=\lim _{s \rightarrow t} D_{h} f^{(\varepsilon)}(s, t)
$$

We consider the random variables $\left\{\xi_{s, t}^{(1,1)}(u)\right\}$ for $(s, t) \in \Gamma_{T}(s, t)$ and $0 \leq u \leq s \leq$ $t \leq T$ satisfying the stochastic integral equation when $\varepsilon=1$,

$$
\begin{aligned}
\xi_{s, t}^{(\varepsilon, 1)}(u)= & \varepsilon^{2} \int_{u}^{s} \partial \sigma\left(f^{(\varepsilon)}(v, t), v, t\right) \xi_{v, t}^{(\varepsilon, 1)}(u) \\
& \times \int_{v}^{t} \sigma\left(f^{(\varepsilon)}(v, y), v, y\right) d y d v \\
& +\varepsilon^{2} \int_{u}^{s} \sigma\left(f^{(\varepsilon)}(v, t), v, t\right) \\
& \times \int_{v}^{t} \partial \sigma\left(f^{(\varepsilon)}(v, y), v, y\right) \xi_{v, y}^{(\varepsilon, 1)}(u) d y d v \\
& +\varepsilon \int_{u}^{s} \partial \sigma\left(f^{(\varepsilon)}(v, t), v, t\right) \xi_{v, t}^{(\varepsilon, 1)}(u) d w_{v} \\
& +\sigma\left(f^{(\varepsilon)}(u, t), u, t\right) .
\end{aligned}
$$


Then we can show that

$$
\int_{0}^{s} \xi_{s, t}^{(1,1)}(u) \dot{h}_{u} d u=D_{h} f^{(1)}(s, t) .
$$

In order to examine the existence of moments of $\left\{\xi_{s, t}^{(1,1)}(u)\right\}$ and other related random variables, we need the following inequality, whose proof is given in Kunitomo and Takahashi (2001).

LEMMA A.4. Suppose for $k_{0}>0, k_{1}>0, A_{N}>0$ and $0<s \leq t \leq T$, a function $w_{N}(u, s, t)$ satisfies (i) $0<w_{N}(u, s, t) \leq A_{N}$ and (ii):

$$
w_{N}(u, s, t) \leq k_{0}+k_{1}\left[\int_{u}^{s} w_{N}(u, v, t) d v+\int_{u}^{s} \int_{v}^{t} w_{N}(u, v, y) d y d v\right] .
$$

Then

$$
w_{N}(u, s, t) \leq k_{0} e^{k_{1}(1+t) s} .
$$

As an illustration of our method based on Lemma A.4, we consider the truncated random variable

$$
\zeta_{s, t}^{N}(u)=\left[\xi_{s, t}^{(1,1)}(u)\right] I_{N}(s, t),
$$

where $I_{N}(s, t)=1$ if $\sup _{0 \leq v \leq s, v \leq y \leq t}\left|\xi_{v, y}(u)\right| \leq N$ and $I_{N}(s, t)=0$ otherwise. By using the boundedness conditions in Assumption II and $\dot{h}_{s}$ being square integrable, we can show that there exist positive constants $M_{i}(i=5, \ldots, 8)$ such that

$$
\begin{aligned}
\left|\zeta_{s, t}^{N}(u)\right|^{p} \leq & M_{5} \int_{u}^{s}\left|\zeta_{v, t}^{N}(u)\right|^{p} d v+M_{6}\left|\int_{u}^{s} \zeta_{v, t}^{N}(u) d w_{v}\right|^{p} \\
& +M_{7} \int_{u}^{s} \int_{v}^{t}\left|\zeta_{v, y}^{N}(u)\right|^{p} d y d v+M_{8}\left|\sigma\left(f^{(1)}(u, t), u, t\right)\right|^{p}
\end{aligned}
$$

By using the martingale inequality, the expectation of the second term is less than $M_{6} \mathbf{E}\left[\int_{u}^{s}\left|\zeta_{v, t}^{N}(u)\right|^{p} d v\right]$. Also the last term in (A.13) is bounded because $\sigma(\cdot)$ is bounded. If we set $w_{N}(u, s, t)=\mathbf{E}\left[\left|\zeta_{s, t}(u)\right|^{p}\right]$, then we can directly apply Lemma A.4. By taking the limit of the expectation function $w_{N}(u, s, t)$ as $N \rightarrow \infty$, we have $\mathbf{E}\left[\left|\xi_{s, t}^{(1,1)}(u)\right|^{p}\right]<+\infty$. By using similar arguments, we have the existence of moments as summarized in the next lemma.

LEMMA A.5. Under Assumption II, for any $p>1$ and $0 \leq u \leq s \leq t \leq T$, we have $\mathbf{E}\left[\sup _{0 \leq u \leq s}\left|\xi_{s, t}^{(1,1)}(u)\right|^{p}\right]<+\infty$ and $\mathbf{E}\left[\sup _{0 \leq u \leq s}\left|f^{(1)}(u, t)\right|^{p}\right]<\infty$.

Using Lemma A.5 and the equivalence of two norms stated in Section 2.1, we now have established the following property of the first-order $H$-derivative,

$$
f^{(1)}(s, t) \in \bigcap_{1<p<+\infty} \boldsymbol{D}_{p}^{1}(\boldsymbol{R}) .
$$


Since we have completed the investigation of the first-order $H$-derivative, our next task is to investigate some properties of the higher order $H$-derivatives of $f^{(1)}(s, t)$. We shall use the induction argument and assume that $f^{(1)}(s, t) \in$ $\bigcap_{1<p<+\infty} \boldsymbol{D}_{p}^{k}(\boldsymbol{R})(k \geq 1)$. Then we have

$$
\begin{aligned}
D_{h}\left[D^{k} f^{(1)}(s, t)\right] & \\
= & \int_{0}^{s}\left[\partial \sigma\left(f^{(1)}(v, t), v, t\right) \int_{v}^{t} \sigma\left(f^{(1)}(v, y), v, y\right) d y D_{h}\left[D^{k} f^{(1)}(v, t)\right]\right] d v \\
+ & \int_{0}^{s}\left[\sigma\left(f^{(1)}(v, t), v, t\right) \int_{v}^{t} \partial \sigma\left(f^{(1)}(v, y), v, y\right)\right. \\
& \left.\times D_{h}\left[D^{k} f^{(1)}(v, y)\right] d y\right] d v \\
+ & \int_{0}^{s} \partial \sigma\left(f^{(1)}(v, t), v, t\right) D_{h}\left[D^{k} f^{(1)}(v, t)\right] d w(v) \\
+ & \int_{0}^{s}\left[G _ { 1 } ^ { ( k ) } \left(\partial^{l} \sigma\left(f^{(1)}(v, t), v, t\right),\right.\right. \\
& \left.D^{l} f^{(1)}(v, t), D_{h} f^{(1)}(v, t), v, t ; l=0, \ldots, k\right) \\
& \times \int_{v}^{t} H_{1}^{(k)}\left(\partial^{l} \sigma\left(f^{(1)}(v, y), v, y\right),\right. \\
& \left.\left.D^{l} f^{(1)}(v, y), D_{h} f^{(1)}(v, y), v, y ; l=0, \ldots, k\right) d y\right] d v \\
+ & \int_{0}^{s}\left[G _ { 2 } ^ { ( k ) } \left(\partial^{l} \sigma\left(f^{(1)}(v, t), v, t\right),\right.\right. \\
& \left.\left.D^{l} f^{(1)}(v, t), D_{h} f^{(1)}(v, t), v, t ; l=0, \ldots, k\right)\right] d w_{v} \\
+ & \int_{0}^{s}\left[G _ { 3 } ^ { ( k ) } \left(\partial^{l} \sigma\left(f^{(1)}(v, t), v, t\right),\right.\right. \\
\left.\left.D^{l} f^{(1)}(v, t), D_{h} f^{(1)}(v, t), v, t ; l=0, \ldots, k\right)\right] d v & \left.\left.D^{l} f^{(1)}(v, t), D_{h} f^{(1)}(v, t), v, t ; l=0, \ldots, k\right)\right] \dot{h}_{v} d v
\end{aligned}
$$

where $H_{1}^{(k)}(\cdot)$ and $G_{j}^{(k)}(\cdot)(j=1, \ldots, 4)$ are defined recursively and they are actually a sequence of polynomial functions. Although the above stochastic integral equation has many terms, the basic structure is the same as the first-order 
$H$-derivative of $f^{(1)}(s, t)$. Now we define the random variables $\left\{\xi_{s, t}^{(1, k)}(u)(k \geq 1)\right\}$ for $0 \leq u \leq s \leq t \leq T$ by

$$
\begin{aligned}
& \xi_{s, t}^{(1, k+1)}(u) \\
& =\int_{0}^{s}\left[\partial \sigma\left(f^{(1)}(v, t), v, t\right) \int_{v}^{t} \sigma\left(f^{(1)}(v, y), v, y\right) d y \xi_{v, t}^{(1, k+1)}(u)\right] d v \\
& +\int_{0}^{s}\left[\sigma\left(f^{(1)}(v, t), v, t\right) \int_{v}^{t} \partial \sigma\left(f^{(1)}(v, y), v, y\right) \xi_{v, y}^{(1, k+1)}(u) d y\right] d v \\
& +\int_{0}^{s} \partial \sigma\left(f^{(1)}(v, t), v, t\right) \xi_{v, t}^{(1, k+1)}(u) d w_{v} \\
& +\int_{0}^{s}\left[G _ { 1 } ^ { ( k ) } \left(\partial^{l} \sigma\left(f^{(1)}(v, t), v, t\right),\right.\right. \\
& \left.D^{l} f^{(1)}(v, t), \xi_{v, t}^{(1, l)}(u), v, t ; l=0, \ldots, k\right) \\
& \times \int_{v}^{t} H_{1}^{(k)}\left(\partial^{l} \sigma\left(f^{(1)}(v, y), v, y\right),\right. \\
& \left.\left.D^{l} f^{(1)}(v, y), \xi_{v, y}^{(1, l)}(u), v, y ; l=0, \ldots, k\right) d y\right] d v \\
& +\int_{0}^{s}\left[G _ { 2 } ^ { ( k ) } \left(\partial^{l} \sigma\left(f^{(1)}(v, t), v, t\right),\right.\right. \\
& \left.\left.D^{l} f^{(1)}(v, t), \xi_{v, t}^{(1, l)}(u), v, t ; l=0, \ldots, k\right)\right] d w_{v} \\
& +\int_{0}^{s}\left[G _ { 3 } ^ { ( k ) } \left(\partial^{l} \sigma\left(f^{(1)}(v, t), v, t\right),\right.\right. \\
& \left.\left.D^{l} f^{(1)}(v, t), \xi_{v, t}^{(1, l)}(u), v, t ; l=0, \ldots, k\right)\right] d v \\
& +\int_{0}^{s}\left[G _ { 4 } ^ { ( k ) } \left(\partial^{l} \sigma\left(f^{(1)}(v, t), v, t\right),\right.\right. \\
& \left.\left.D^{l} f^{(1)}(v, t), \xi_{v, t}^{(1, l)}(u), v, t ; l=0, \ldots, k\right)\right] d v .
\end{aligned}
$$

Then we can show that

$$
\int_{0}^{s} \xi_{s, t}^{(1, k+1)}(u) \dot{h}_{u} d u=D_{h}\left[D^{k} f^{(1)}(s, t)\right] .
$$

From the above representation, we have

$$
\left|D^{k+1} f^{(1)}(s, t)\right|_{H^{\otimes(k+1)}}^{2}=\int_{0}^{s}\left|\xi_{s, t}^{(1, k+1)}(u)\right|_{H^{\otimes k}}^{2} d u .
$$


Applying Lemma A.4, repeating the procedure as Lemma A.5 and using induction with respect to $k$, we have for any integers $k \geq 1$ and $p>1$,

$$
\mathbf{E}\left[\left|\xi_{s, t}^{(1, k)}(u)\right|_{H^{\otimes(k-1)}}^{p}\right]<+\infty .
$$

Then by the same construction and induction arguments, for positive integers $k(\geq 1)$ we can define a sequence of random variables $\left\{f^{(\varepsilon)}(s, t)\right\},\left\{\xi_{s, t}^{(\varepsilon, k)}(u)\right\}$ and $\left\{D^{k} f^{(\varepsilon)}(s, t)\right\}$. Hence we have completed the proof of Theorem 4.2.

Acknowledgments. This paper is a revised version of Discussion Paper No. 98-F-6 at the Faculty of Economics, University of Tokyo. We thank Professors S. Kusuoka and N. Yoshida for some discussions on technical issues involved. We also thank a referee and an Associate Editor for their helpful comments on earlier versions. However, we are responsible for any remaining errors in this paper.

\section{REFERENCES}

Duffie, D. (1996). Dynamic Asset Pricing Theory, 2nd ed. Princeton Univ. Press.

Fujikoshi, Y., Morimune, K., Kunitomo, N. and Taniguchi, M. (1982). Asymptotic expansions of the distributions of the estimates of coefficients in a simultaneous equation system. J. Econometrics 18 191-205.

HE, H. and TAKAHASHI, A. (2000). A variable reduction technique for pricing average-rate options. Internat. Rev. Finance 1 123-142.

HeAth, D., JARRow, R. and MORTON, A. (1992). Bond pricing and the term structure of interest rates: A new methodology for contingent claims valuation. Econometrica 60 77-105.

IKeda, N. and Watanabe, S. (1989). Stochastic Differential Equations and Diffusion Processes, 2nd ed. North-Holland, Amsterdam.

Kim, Y. and KUnitomo, N. (1999). Pricing options under stochastic interest rates: A new approach. Asia-Pacific Financial Markets 6 49-70.

Kunitomo, N. and Kim, Y. (2001). Effects of stochastic interest rates and volatility on contingent claims. Discussion Paper CIRJE-F-129, Faculty of Economics, Univ. Tokyo.

Kunitomo, N. and TaKahashi, A. (1992). Pricing average options. Japan Financial Review 14 1-20 (in Japanese).

Kunitomo, N. and TAKAHASHI, A. (1995). The asymptotic expansion approach to the valuation of interest rate contingent claims. Discussion Paper 95-F-19, Faculty of Economics, Univ. Tokyo.

KUnitomo, N. and TAKAHASHI, A. (1998). On validity of the asymptotic expansion approach in contingent claim analysis. Discussion Paper 98-F-6, Faculty of Economics, Univ. Tokyo.

Kunitomo, N. and TAKAHASHI, A. (2001). The asymptotic expansion approach to the valuation of interest rate contingent claims. Math. Finance 11 117-151.

Kusuoka, S. and Stroock, D. (1982). Applications of the Malliavin calculus. I. Taniguchi Symposium, SA Katata 271-306.

Morton, A. J. (1989). Arbitrage and martingales. Ph. D. dissertation, Cornell Univ.

Nualart, D. (1995). Malliavin Calculus and the Related Topics. Springer, New York.

SHIGEKAWA, I. (1998). Stochastic Analysis. Iwanami-Shoten (in Japanese).

SøREnSEn, M. and YoshidA, N. (2000). Random limit expansion for small diffusion processes. Unpublished manuscript.

Stroock, D. and Varadhan, S. (1979). Multidimensional Diffusion Processes. Springer, New York. 
TAKAHASHI, A. (1995). Essays on the valuation problems of contingent claims. Ph.D. dissertation, Univ. California, Berkeley.

TAKAhashi, A. (1999). An asymptotic expansion approach to pricing contingent claims. AsiaPasific Financial Markets 6 115-151.

TAKahashi, A. and Yoshida, N. (2001). Asymptotic expansion scheme for the optimal portfolio for investment. Preprint.

Watanabe, S. (1984). Lectures on Stochastic Differential Equations and Malliavin Calculus. Springer, New York.

Watanabe, S. (1987). Analysis of Wiener functionals (Malliavin calculus) and its applications to heat kernels. Ann. Probab. 15 1-39.

YoshidA, N. (1992). Asymptotic expansions of maximum likelihood estimators for small diffusions via the theory of Malliavin-Watanabe. Probab. Theory Related Fields 92 275-311.

Yoshida, N. (1997). Asymptotic expansions for martingales on Wiener space and applications to statistics. Probab. Theory Related Fields 109 301-342.

FACULTY OF ECONOMICS

UNIVERSITY OF TOKYO

BUNKYO-KU, HONGO 7-3-1

TOKYO 113

JAPAN

\author{
GRADUATE SCHOOL \\ OF MATHEMATICAL SCIENCES \\ UNIVERSITY OF TOKYO \\ MEguro-KU, KomABA 3-8-1 \\ TOKYO 153 \\ JAPAN
}

